\title{
Electrostatic Force Distribution on an Electrodynamic Screen
}

\author{
Arash Sayyaha,*, Mark N. Horenstein $^{\mathrm{a}}$, Malay K. Mazumder ${ }^{\mathrm{a}}$, Goodarz Ahmadi ${ }^{\mathrm{b}}$ \\ ${ }^{a}$ Department of Electrical and Computer Engineering, Boston University, Boston, MA 02215 \\ ${ }^{b}$ Department of Mechanical and Aeronautical Engineering, Clarkson University, Potsdam, NY 13699
}

\begin{abstract}
The enormous potential of the solar energy harvesting plants for providing clean energy is severely limited by dust accumulation on their optical surfaces. In lieu of the most commonly-practiced manual cleaning method of using high-pressure water jets, the electrodynamic screen (EDS) technology offers an attractive solution for removing dust particles from optical surfaces using electrostatic forces. In this paper, the impacts of different EDS design parameters in the electric field distribution on an EDS have been studied. Furthermore, based on electric field expressions, closed-form solutions for multipolar dielectrophoretic (DEP) forces in the EDS application are provided. Detailed evaluation of the EDS performance necessitates investigation of different forces involved in dust removal process. We have provided different comparisons between repelling and attracting forces exerted on dust particles in the dust removal process. Comparison results elucidate how EDS performs in removing a given size range of dust particles. The significant detrimental impact of relative humidity in the EDS performance has been quantitatively addressed. It is shown how just $10 \%$ increase in relative humidity can make the repelling force ineffective in dust removal process.
\end{abstract}

Keywords: Adhesion forces, dielectrophoresis, dust removal, electrodynamic screen (EDS), electrostatics, Laplace's equation

\section{Introduction}

In spite of the enormous potential of the solar energy harvesting plants for providing clean energy 3 with minimal carbon footprint, dust accumulation on their optical surfaces, the so-called "soiling" effect 4 is a severe performance-limiting factor that decreases the efficiency by absorption and scattering of the

\footnotetext{
${ }^{*}$ Corresponding author

Email address: arashs@bu . edu (Arash Sayyah)
} 
2010impact 75

6

incident light [1, 2, 3]. The performance drop could happen gradually in the course of exposure time, attributed to the accumulation of atmospheric dust or abruptly due to a dust storm, in which the efficiency loss can reach $60 \%$ in less than 6 hours [4].

In order to mitigate the soiling losses in solar collectors, using high-pressure water jets, often mixed with detergents, is considered the most commonly-practiced method in large-scale solar plants [5, 6, 7]. Scarcity of water resources, large amounts of water usage, deionization process expenses, and the labor cost to perform the cleaning procedure are the main prohibitive factors associated with the cleaning method using water. As an alternative method, robotic devices are under developmental stages to be utilized in large-scale solar plants to restore their efficiency [8, 9, 10]. Although significant endeavors have been pursuit in improving the performance of robotic devices, they are still facing some challenges such as scalibility, minimization of water usage, maintenance and operation costs, to name a few. As an alternative, the concept of automatic dust removal using the electrostatic forces of electrodynamic screen (EDS) technology is in a developmental stage and on its way toward commercialization [11, 12, 13, 14, 15, 16, 17, 18, 19, 20]. The EDS consists of a set of parallel electrodes, either transparent or opaque, deposited on a glass substrate and embedded by a thin transparent dielectric film. When a three-phase, low frequency $(5-10 \mathrm{~Hz})$, high voltage-pulses are applied to the electrodes, accumulated dust particles on the EDS surface are charged electrostatically and repelled by electrostatic forces.

In dust particle removal by the EDS, Coulomb force $q \mathbf{E}$ is considered the predominant force among the repelling forces. Therefore, having a detailed model of the electrostatic force distribution on an electrodynamic screen is of utmost importance in evaluating its performance, examination of influential parameters, and maximizing dust removal efficiency. In this paper, we present analytical solutions for the electric field distribution in an EDS configuration with one layer of dielectric coating. The impacts of influential parameters: electrode width, inter-electrode spacing, thickness and relative permittivity of the dielectric coating in the electric field distribution have been studied thoroughly.

Since the electric field distribution generated by the EDS is non-uniform, the accumulated dust particles, whether charged or neutral, experience dielectrophoretic (DEP) forces [21]. Numerous studies have pursuit the application of dielectrophoresis phenomenon in the biological research areas and microfluidic devices for particle manipulation and separation [22, 23, 24, 25]. This phenomenon, however, has not been studied thoroughly in the EDS application. It is strongly speculated that the dust particles are charged electrostatically through triboelectrification process caused by the DEP forces, especially in ini- 
tial moments of EDS operation when the particles have no net charge or their charge is insignificant [26]. In this study, the analytical expressions for the mulipolar DEP forces up to the third-order are derived, based upon the closed-form solutions of the electric field distribution. Our analytical expressions for the multipolar DEP forces enable the study of the impact of EDS design parameters on these forces. The analytical expressions for the DEP forces presented herein can be embedded efficiently in the equation of motion for particle trajectory modeling, something that seemed a formidable task previously [27].

The ultimate goal of the EDS activation is to ensure at each given point over the EDS surface, the summation of repelling forces are greater than the attracting ones in the balance of forces exerted on the particle. In other words, to levitate a particle from the EDS surface, the exerted Coulomb force should dominate all the other attracting forces that push the particle towards the EDS surface. To evaluate the van der Waals force and capillary force and compare them with other forces, we have initially used the ideal models suggested in the literature when a spherical dust particle is in contact with a surface, both being perfectly smooth with no surface asperities. Idealized models not only have resulted in overestimation of the adhesion forces in the comparison, but also do not comply with the experimental observations we have had for the EDS performance. To have a more detailed and realistic comparison, we have considered the surface asperities of the top EDS surface into the adhesion force model. Furthermore, the significant role of relative humidity in the increase of capillary force and consequently inferior performance of the EDS in dust removal process has been addressed.

\section{Analytical Solutions}

\subsection{Electric Field Distribution}

Figure 1 shows the cross section of an EDS configuration with one transparent dielectric coating on top of the electrodes. In Fig. 1, the thickness of the dielectric layer and its relative permittivity are denoted as $\delta$ and $\epsilon_{d}$, respectively. Also, the width of the electrodes and inter-electrode spacing are denoted as $w$ and $g$, respectively. It is assumed that the height of the electrodes is negligible compared to the thickness of the dielectric layer. For a three-phase activated EDS, the fundamental spatial period denoted as $T_{s}$ is written as:

$$
T_{s}=3(w+g) .
$$


Since we assume there is no net charge in the space above the dielectric layer in Fig.1, i.e. the deposited dust particles do not have a net electric charge initially, we can write Laplace's equation for the electric potential on the EDS surface $\phi(x, y)$ in Cartesian coordinates as:

$$
\frac{\partial \phi^{2}(x, y)}{\partial x^{2}}+\frac{\partial \phi^{2}(x, y)}{\partial y^{2}}=0, \quad 0 \leq x \leq T_{s}, \quad \delta \leq y<\infty
$$

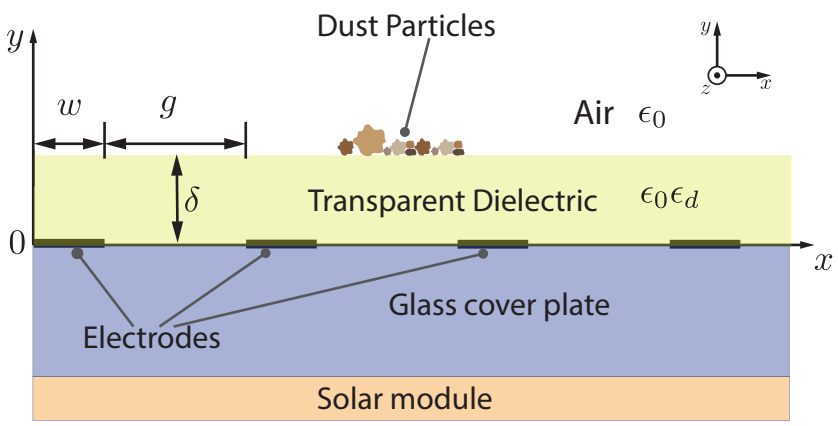

Figure 1: Cross section of the EDS configuration with one layer of transparent dielectric coating on top of the electrodes with thickness $\delta$ and relative permittivity of $\epsilon_{d}$. The problem is assumed to be infinite in the $z$ direction (normal to the page). The solar module represents a photovoltaic (PV) cell or a reflecting mirror in a concentrated solar power (CSP) system.

electric potential at $y=0$ can be expressed in terms of Fourier series as

$$
\phi(x, 0)=\sum_{k=0}^{\infty} a_{k} \cos \left(\Omega_{0} k x\right)+b_{k} \sin \left(\Omega_{0} k x\right) .
$$

Sayyah et al. [28] have provided the comprehensive analytical expressions for electric field distributions in an EDS with two stacked layers of dielectric coatings. Following the same approach, we obtain the closed-form solutions for the electric field distributions in the dielectric layer and on the EDS surface of Fig. 1. By denoting unit vectors in $x$ and $y$ directions as $\mathbf{a}_{x}$ and $\mathbf{a}_{y}$, respectively, the electric field in the dielectric layer $\mathbf{E}_{d}$ and in the air (i.e. $y \geq \delta$ ) $\mathbf{E}_{a}$ are written as: 


$$
\begin{aligned}
\mathbf{E}_{d}(x, y)= & \Omega_{0}\left\{\sum_{k} \frac{\sinh \left[k \Omega_{0}(y-\delta)\right]}{\sinh \left(k \Omega_{0} \delta\right)}\left[-k a_{k} \sin \left(\Omega_{0} k x\right)+k b_{k} \cos \left(\Omega_{0} k x\right)\right]\right. \\
& \left.+\sum_{k} \frac{\sinh \left(k \Omega_{0} y\right)}{\sinh \left(k \Omega_{0} \delta\right)}\left[k g_{k} \sin \left(\Omega_{0} k x\right)-k h_{k} \cos \left(\Omega_{0} k x\right)\right]\right\} \mathbf{a}_{x}+ \\
& \Omega_{0}\left\{\sum_{k} \frac{\cosh \left[k \Omega_{0}(y-\delta)\right]}{\sinh \left(k \Omega_{0} \delta\right)}\left[k a_{k} \cos \left(\Omega_{0} k x\right)+k b_{k} \sin \left(\Omega_{0} k x\right)\right]\right. \\
& \left.-\sum_{k} \frac{\cosh \left(k \Omega_{0} y\right)}{\sinh \left(k \Omega_{0} \delta\right)}\left[k g_{k} \cos \left(\Omega_{0} k x\right)+k h_{k} \sin \left(\Omega_{0} k x\right)\right]\right\} \mathbf{a}_{y}, \\
\mathbf{E}_{a}(x, y)= & \left\{\Omega_{0} \sum_{k} e^{-\Omega_{0} k(y-\delta)}\left[k g_{k} \sin \left(\Omega_{0} k x\right)-k h_{k} \cos \left(\Omega_{0} k x\right)\right]\right\} \mathbf{a}_{x}+ \\
& \left\{\Omega_{0} \sum_{k} e^{-\Omega_{0} k(y-\delta)}\left[k g_{k} \cos \left(\Omega_{0} k x\right)+k h_{k} \sin \left(\Omega_{0} k x\right)\right]\right\} \mathbf{a}_{y},
\end{aligned}
$$

in which the Fourier coefficients $g_{k}$ and $h_{k}$ are obtained from Eqs. 6 and 7, respectively, assuming the Fourier coefficients $a_{k}$ and $b_{k}$ are provided:

$$
\begin{aligned}
g_{k} & =\frac{\epsilon_{d}}{\sinh \left(k \Omega_{0} \delta\right)+\epsilon_{d} \cosh \left(k \Omega_{0} \delta\right)} a_{k} \\
h_{k} & =\frac{\epsilon_{d}}{\sinh \left(k \Omega_{0} \delta\right)+\epsilon_{d} \cosh \left(k \Omega_{0} \delta\right)} b_{k} .
\end{aligned}
$$

As can be seen in Eq. 5, the electric field distribution is dependent upon thickness of the dielectric layer $\delta$, electrode width and inter-electrode spacing since $\Omega_{0}=\frac{2 \pi}{3(w+g)}$, and Fourier coefficients $g_{k}$ and $h_{k}$. As provided in Eqs. 6 and 7, the Fourier scoefficients $g_{k}$ and $h_{k}$ are both functions of relative permittivity of the dielectric layer. In Section 3, we corroborate the analytical expressions for the electric field distribution using $\mathrm{COMSOL}^{\circledR}$ Multiphysics ${ }^{\circledR}$ software. Furthermore, we investigate the impacts of the electrode width, inter-electrode spacing, thickness and relative permittivity of the dielectric coating on the electric field distribution on the EDS surface. 


\subsection{Multipolar Dielectrophoretic (DEP) Forces}

As noted in preceding section, the electric field distribution on the EDS surface is a nonuniform electric field. When a dust particle, either neutral or charged, approaches the EDS proximity or simply is deposited on the EDS surface, it is exposed to the applied nonuniform electric field present in the space above the EDS. In this exposure, electric charges are redistributed differently at the two interfaces of the particle and the medium (air). This charge at the interface of particle and medium is called Maxwell-Wagner interfacial charge [29]. The amount of charge accumulated at the interface depends upon the electrical properties (conductivity and permittivity) of both the particle and medium and the applied electric field. Due to this charge accumulation on the two sides of the particle, a net dipole forms across the particle, which its direction either parallel or against the electric field depends on the polarizability of the particle. The induced dipole experiences different strengths of the electric field at its both ends because of the nonuniformity of the field and this imbalance of forces gives rise to the particle movement. This is called the dielectrophoresis (DEP) effect. Dielectrophoresis have been used widely in microsystems as an effective method for manipulating particles. However, as mentioned in the introduction of the paper, it has not received significant attention in the EDS application. Since we have derived the analytical expressions for the electric field distribution, the calculation of DEP forces has become feasible.

The general n-order multipolar DEP force for a 2-D problem is given by [30]:

$$
\mathbf{F}^{(n)}=-\frac{4 \pi n \epsilon_{m} K_{n} R^{2 n+1}}{(2 n-1) ! !} \sum_{p+q=n} \frac{1}{p ! q !}\left[\frac{\partial^{n} \phi}{\partial x^{p} \partial y^{q}} \frac{\partial^{n} \mathbf{E}}{\partial x^{p} \partial y^{q}}\right]
$$

in which $R$ is the particle radius, $\phi$ is the electrostatic potential in the space in the absence of particle, $\mathbf{E}$ is the applied electric field, $\epsilon_{m}$ is the dielectric permittivity of the medium, and $K_{n}$ is the generalized polarization coefficient. The general expression for the polarization coefficient is given by

$$
K_{n}(\omega)=\frac{\epsilon_{p}^{*}(\omega)-\epsilon_{m}^{*}(\omega)}{n \epsilon_{p}^{*}(\omega)+(n+1) \epsilon_{m}^{*}(\omega)},
$$

where $\epsilon_{m}^{*}$ and $\epsilon_{p}^{*}$ are the complex dielectric permittivity of the medium and particle, respectively, and $\omega$ is the angular frequency of the applied electric field. Complex electrical permittivity of a material 
denoted as $\epsilon^{*}$ in general form is written as

$$
\epsilon^{*}=\epsilon-\mathbf{i} \sigma / \omega
$$

in which $\mathbf{i}$ and $\sigma$ are the imaginary unit and the conductivity of the material, respectively. At low and high frequency, the polarization coefficient is reduced to the following expressions, respectively:

$$
\begin{aligned}
K_{n}(\omega) & =\frac{\sigma_{p}-\sigma_{m}}{n \sigma_{p}+(n+1) \sigma_{m}} \text { when } \omega \rightarrow 0, \\
K_{n}(\omega) & =\frac{\epsilon_{p}-\epsilon_{m}}{n \epsilon_{p}+(n+1) \epsilon_{m}} \text { when } \omega \rightarrow \infty .
\end{aligned}
$$

At low frequencies of the applied electric field, the conductivity of the particle and medium determine the polarization coefficient while the dielectric permittivities of the two materials are the influential parameters at high frequencies. If both materials, i.e. dust particle and the medium (air) are perfect dielectric materials, then their conductivities in Eq. 9 can be considered to be zero. Hence, the general polarization coefficient, provided in Eq. 9, is reduced to the following expression:

$$
K_{n}=\frac{\epsilon_{p}-\epsilon_{m}}{n \epsilon_{p}+(n+1) \epsilon_{m}}
$$

which is independent of the angular frequency of the applied electric field. Henceforth in this paper, we assume the dust particles and the air are both perfect dielectric materials and the polarization coefficient is obtained from Eq. 13. Since the polarizability of the particles in the EDS application are greater than the air: $\epsilon_{p}>\epsilon_{0}$, the polarization coefficient is always positive and the dust particles experience positive dielectrophoresis and move towards the regions with higher electric field intensities. Therefore, if the particle is assumed to be suspended in the space above the EDS surface, mulipolar DEP forces try to push the particle towards EDS surface, i.e. $y=\delta$. First-order polarization coefficient $K_{1}$ is generally called the Clausius-Mossotti factor. In this paper, we consider the DEP forces for a lossless spherical dielectric particle with radius $R$ and relative permittivity $\epsilon_{p}$ suspended in a lossless, dielectric medium of permittivity $\epsilon_{m}$ which is equal to $\epsilon_{0}$. Particles with ohmic losses are not in the scope of this work [31].

Figure 2(a) shows a dust particle deposited on an EDS surface and Fig. 2(b) depicts the charge accumulation on two sides of the dust particle in a non-uniform electric field, where the dust particle 


\section{a}

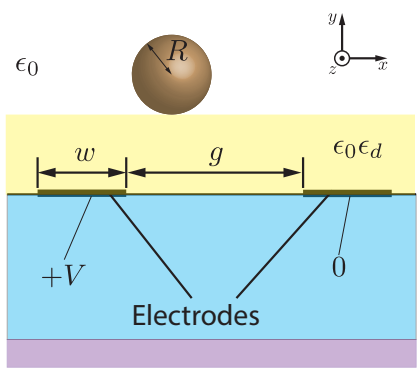

b

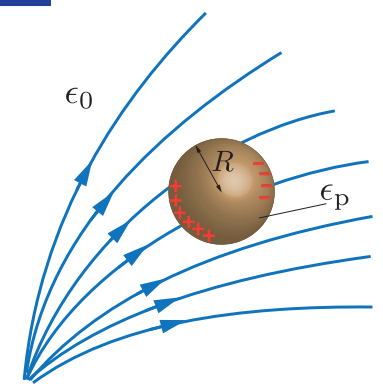

Figure 2: (a) Dust particle deposited on an EDS surface, where one of the electrodes is connected to a positive voltage $+V$ and the other electrode is at 0 potential and (b) dust particle with radius $R$ and permittivity $\epsilon_{p}$, exposed to the non-uniform electric field generated by EDS, experiencing positive DEP force. Accumulation of positive electric charges on the left side of the particle, where electric field lines are closer, i.e. stronger electric field intensity, is higher than the right side of particle, where negative electric charges have accumulated due to a weaker electric field intensity.

The total DEP force exerted on the particle is given by

$$
\mathbf{F}^{\mathrm{tot}}=\mathbf{F}^{(1)}+\mathbf{F}^{(2)}+\mathbf{F}^{(3)}+\ldots
$$

$$
\begin{aligned}
\mathbf{F}^{(1)} & =4 \pi \epsilon_{0} R^{3} K_{1} \mathbf{S}^{(1)}, \\
\mathbf{F}^{(2)} & =\frac{4}{3} \pi \epsilon_{0} R^{5} K_{2} \mathbf{S}^{(2)}, \\
\mathbf{F}^{(3)} & =\frac{2}{15} \pi \epsilon_{0} R^{7} K_{3} \mathbf{S}^{(3)} .
\end{aligned}
$$

The polarization coefficients $K_{1}$ to $K_{3}$ in Eqs. 15 to 17 , according to Eq. 13 , are given by:

$$
\begin{aligned}
& K_{1}=\frac{\epsilon_{p}-\epsilon_{0}}{\epsilon_{p}+2 \epsilon_{0}}, \\
& K_{2}=\frac{\epsilon_{p}-\epsilon_{0}}{2 \epsilon_{p}+3 \epsilon_{0}}, \\
& K_{3}=\frac{\epsilon_{p}-\epsilon_{0}}{3 \epsilon_{p}+4 \epsilon_{0}} .
\end{aligned}
$$


Furthermore, in Eqs. 15 to 17 , the DEP force spatial factors are denoted as $\mathbf{S}^{(n)}$ as they depend upon the gradient operations on the electric field. They are two-dimensional vectors given by

$$
\mathbf{S}^{(n)}=S_{x}^{(n)} \mathbf{a}_{x}+S_{y}^{(n)} \mathbf{a}_{y}
$$

The closed-form expressions for the DEP spatial factors are given by

$$
\begin{aligned}
S_{\ell}^{(1)} & =E_{x} \frac{\partial E_{\ell}}{\partial x}+E_{y} \frac{\partial E_{\ell}}{\partial y}, \\
S_{\ell}^{(2)} & =\frac{\partial E_{x}}{\partial x} \frac{\partial^{2} E_{\ell}}{\partial x^{2}}+2 \frac{\partial E_{x}}{\partial y} \frac{\partial^{2} E_{\ell}}{\partial x \partial y}+\frac{\partial E_{y}}{\partial y} \frac{\partial^{2} E_{\ell}}{\partial y^{2}}, \\
S_{\ell}^{(3)} & =\frac{\partial^{2} E_{x}}{\partial x^{2}} \frac{\partial^{3} E_{\ell}}{\partial x^{3}}+3 \frac{\partial^{2} E_{y}}{\partial x^{2}} \frac{\partial^{3} E_{\ell}}{\partial y \partial x^{2}}+3 \frac{\partial^{2} E_{x}}{\partial y^{2}} \frac{\partial^{3} E_{\ell}}{\partial x \partial y^{2}}+\frac{\partial^{2} E_{y}}{\partial y^{2}} \frac{\partial^{3} E_{\ell}}{\partial y^{3}},
\end{aligned}
$$

where $\ell=x$ - or $y$-direction, respectively. It should be noted that since the curl of the electrostatic field is zero $(\nabla \times \mathbf{E}=0)$, we have $\partial E_{y} / \partial x=\partial E_{x} / \partial y$. This point has been used in the simplification of the expressions provided in Eqs. 22 to 24

In previous section, we derived the analytical expressions for the electric field components in the air, i.e. Eq. 5. Based upon Eq. 5, the explicit expressions for the DEP spatial factors $\mathbf{S}^{(1)}$ to $\mathbf{S}^{(3)}$ can be derived by taking the partial derivation with respect to $x$ and $y$. The first-order DEP spatial factors are:

$$
\begin{aligned}
& S_{x}^{(1)}=\Omega_{0}^{3} \sum_{j=0}^{\infty} \sum_{k=0}^{j} k(j-k)^{2} e^{-j \Omega_{0}(y-\delta)}\left[C_{1} \cos \left(\Omega_{0}(j-k) x\right)+C_{2} \sin \left(\Omega_{0}(j-k) x\right)\right], \\
& S_{y}^{(1)}=\Omega_{0}^{3} \sum_{j=0}^{\infty} \sum_{k=0}^{j} k(j-k)^{2} e^{-j \Omega_{0}(y-\delta)}\left[C_{2} \cos \left(\Omega_{0}(j-k) x\right)-C_{1} \sin \left(\Omega_{0}(j-k) x\right)\right],
\end{aligned}
$$

The second-order DEP spatial factors are given by:

$$
\begin{aligned}
& S_{x}^{(2)}=2 \Omega_{0}^{5} \sum_{j=0}^{\infty} \sum_{k=0}^{j} k^{2}(j-k)^{3} e^{-j \Omega_{0}(y-\delta)}\left[C_{1} \cos \left(\Omega_{0}(j-k) x\right)+C_{2} \sin \left(\Omega_{0}(j-k) x\right)\right], \\
& S_{y}^{(2)}=2 \Omega_{0}^{5} \sum_{j=0}^{\infty} \sum_{k=0}^{j} k^{2}(j-k)^{3} e^{-j \Omega_{0}(y-\delta)}\left[C_{2} \cos \left(\Omega_{0}(j-k) x\right)-C_{1} \sin \left(\Omega_{0}(j-k) x\right)\right],
\end{aligned}
$$


and finally, the third-order DEP spatial factors are as follows:

$$
\begin{aligned}
& S_{x}^{(3)}=4 \Omega_{0}^{7} \sum_{j=0}^{\infty} \sum_{k=0}^{j} k^{3}(j-k)^{4} e^{-j \Omega_{0}(y-\delta)}\left[C_{1} \cos \left(\Omega_{0}(j-k) x\right)+C_{2} \sin \left(\Omega_{0}(j-k) x\right)\right], \\
& S_{y}^{(3)}=4 \Omega_{0}^{7} \sum_{j=0}^{\infty} \sum_{k=0}^{j} k^{3}(j-k)^{4} e^{-j \Omega_{0}(y-\delta)}\left[C_{2} \cos \left(\Omega_{0}(j-k) x\right)-C_{1} \sin \left(\Omega_{0}(j-k) x\right)\right],
\end{aligned}
$$

in which

$$
\begin{aligned}
& C_{1}=\left[g_{k} g_{j-k}+h_{k} h_{j-k}\right] \sin \left(\Omega_{0} k x\right)+\left[g_{k} h_{j-k}-h_{k} g_{j-k}\right] \cos \left(\Omega_{0} k x\right), \\
& C_{2}=\left[g_{k} h_{j-k}-h_{k} g_{j-k}\right] \sin \left(\Omega_{0} k x\right)-\left[g_{k} g_{j-k}+h_{k} h_{j-k}\right] \cos \left(\Omega_{0} k x\right) .
\end{aligned}
$$

The Fourier coefficients $g_{k}$ and $h_{k}$ are obtained from Eqs. 6 and 7, respectively. In deriving the closed-form solutions for the DEP spatial factors, we have used the Cauchy product, which states that if we have two infinite sequences $\sum_{n=0}^{\infty} a_{n}$ and $\sum_{n=0}^{\infty} b_{n}$, then the Cauchy product is defined by a discrete convolution as follows:

$$
\left(\sum_{n=0}^{\infty} a_{n}\right) \cdot\left(\sum_{n=0}^{\infty} b_{n}\right)=\sum_{j=0}^{\infty} c_{j}, \text { where } c_{j}=\sum_{k=0}^{j} a_{k} b_{j-k} .
$$

Now we have comprehensive expressions for the DEP force up to the third-order. These expressions can be easily implemented in the particle trajectory modeling studies for the EDS application. Dependent upon of the geometry of the configuration of the electric field and its degree of non-uniformity, different approximations can be used for the DEP forces.

\section{Numerical Analysis}

\subsection{Numerical Verification of the Analytical Solution}

The solution for the electric field distribution in Section 2 is general and can be used to formulate the problem for a single-phase or multi-phase EDS activation. We assume the EDS in this paper is connected to a three-phase power supply that generates rectangular waveforms with $50 \%$ duty cycle. The voltage waveforms in one time period are shown in Fig. 3 , in which phases A, B, and C form 6 different combination of voltages, numbered 1 through 6 in Fig. 3 


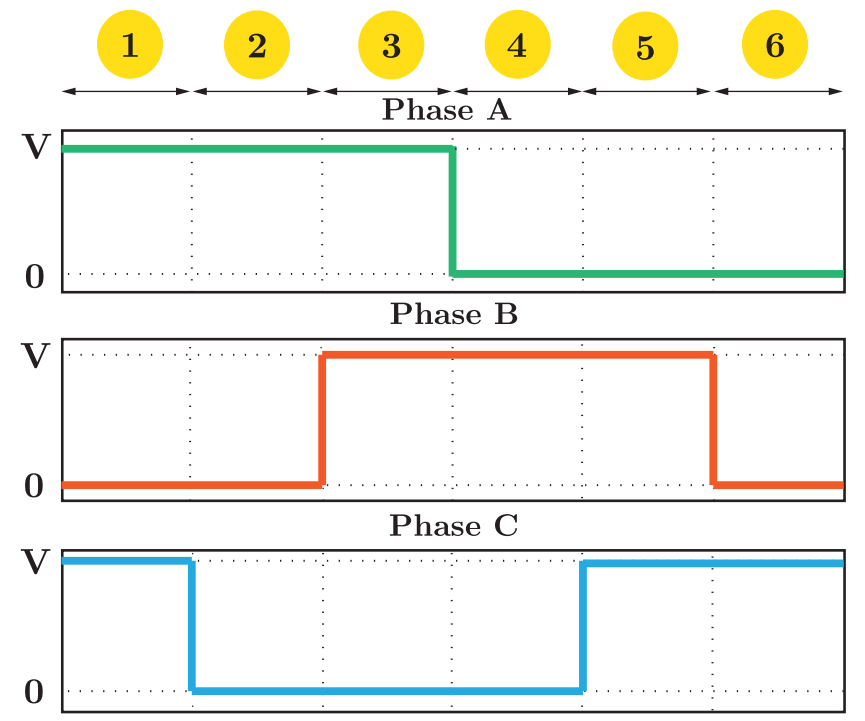

\section{Time $[\mathrm{s}]$}

Figure 3: Three-phase rectangular voltage waveforms with 50\% duty cycle in one time period $T_{t}$.

$$
\begin{aligned}
a_{k} & =\frac{2}{T_{s}} \int_{T_{s}} \phi_{d}(x, 0) \cos (\Omega x) d x \\
b_{k} & =\frac{2}{T_{s}} \int_{T_{s}} \phi_{d}(x, 0) \sin (\Omega x) d x
\end{aligned}
$$

in which $\phi_{d}(x, 0)$ is the electric potential at $y=0$ in Fig. 1 and $\Omega=k \Omega_{0}$. Assuming a linear voltage profile change between adjacent electrodes, the expressions for the Fourier coefficients $a_{k}$ and $b_{k}$ are obtained as follows:

$$
\begin{aligned}
a_{k}= & \frac{1}{\pi g \Omega_{0} k^{2}}\left\{\left(V_{2}-V_{1}\right)\left[\cos \left(k \Omega_{0}(w+g)\right)-\cos \left(k \Omega_{0} w\right)\right]+\right. \\
& \left(V_{3}-V_{2}\right)\left[\cos \left(2 k \Omega_{0}(w+g)\right)-\cos \left(k \Omega_{0}(2 w+g)\right)\right]+\left(V_{1}-V_{3}\right)\left[1-\cos \left(k \Omega_{0}(3 w+2 g)\right)\right](33,6)
\end{aligned}
$$$$
b_{k}=\frac{1}{\pi g \Omega_{0} k^{2}}\left\{\left(V_{2}-V_{1}\right)\left[\sin \left(k \Omega_{0}(w+g)\right)-\sin \left(k \Omega_{0} w\right)\right]+\right.
$$

$$
\left.\left(V_{3}-V_{2}\right)\left[\sin \left(2 k \Omega_{0}(w+g)\right)-\sin \left(k \Omega_{0}(2 w+g)\right)\right]-\left(V_{1}-V_{3}\right) \sin \left(k \Omega_{0}(3 w+2 g)\right)\right\} .
$$

in which $V_{1}, V_{2}$, and $V_{3}$ denote the voltages of phase $\mathrm{A}, \mathrm{B}$, and $\mathrm{C}$ at each time instant, respectively. 
The voltages $V_{1}, V_{2}$, and $V_{3}$ switch between 0 and $V$, as indicated in Fig. 3. Once the Fourier coefficients $a_{k}$ and $b_{k}$ are calculated, coefficients $g_{k}$ and $h_{k}$ are obtained from Eqs. 6 and 7 , correspondingly. It should be re-emphasized that since the voltages $V_{1}, V_{2}$, and $V_{3}$ are functions of time $t$, the Fourier coefficients $a_{k}$ and $b_{k}$ are also functions of time. This point should be implicitly considered in working with the Fourier coefficients.

In order to corroborate the analytical solutions provided in Section 2 for the electric field distribution, the EDS model with one layer of transparent dielectric coating has been developed in the $\mathrm{COMSOL}^{\circledR}$ Multiphysics ${ }^{\circledR}$ finite element analysis (FEA) software. Figure 4 shows the results for the electric potential and electric field norm, i.e. $|\mathbf{E}|=\left(E_{x}^{2}+E_{y}^{2}\right)^{0.5}$, as well as the electric vector field in one fundamental spatial period. The maximum and minimum of electric potential are 974 and 44 volts, respectively, on the EDS surface. As can be expected, the electric field vectors in Fig. 4(b) originate from the energized electrodes (first and third) and terminate in the electrode with zero potential (second electrode). The normal component of electric field $E_{y}$ reaches its maximum value of $2.062 \mathrm{MV} / \mathrm{m}$ on the EDS surface just above the end of the first and start of third electrodes in the period, in which both electrodes are energized with $1 \mathrm{kV}$. The minimum value of the $E y$ is $-2.67 \mathrm{MV} / \mathrm{m}$, right above the tips of the second electrode which is at zero electric potential. The maximum of the electric field norm reaches $3.11 \mathrm{MV} / \mathrm{m}$ at the end of second electrode.

The normal component of the electric field, i.e. $E_{y}$ has been calculated for 6 different cases of the voltage combinations, highlighted in Fig. 4, using the analyical (ANA) solutions provided in Section 2. The ANA results have been compared with the finite element analysis (FEA) results in Fig. 5. The positions of the three electrodes in one fundamental spatial period along the $x$-axis are highlighted with black bars in Fig. 5. To obtain the electric field components from the ANA method, the Fourier coefficients $a_{k}$ and $b_{k}$ were initially calculated for each case of voltage combination from Eqs. 36 and 38 , respectively. Then, Fourier coefficients $g_{k}$ and $h_{k}$ are calculated according to Eqs. 67and 7, respectively. The electric field components on the EDS surface (i.e. $y=\delta$ ) can be calculated using the expression for the electric field distribution in the air as provided in Eq. 5. In Fig. 5, the maximum of $E_{y}$ reaches 3.49 MV/m in cases 1,3 , and 5 while in cases 2, 4, and 6, the maximum of $E_{y}$ is $4.52 \mathrm{MV} / \mathrm{m}$. As can be seen, the analytical results are in very good agreement with the numerical simulation results. In order to 


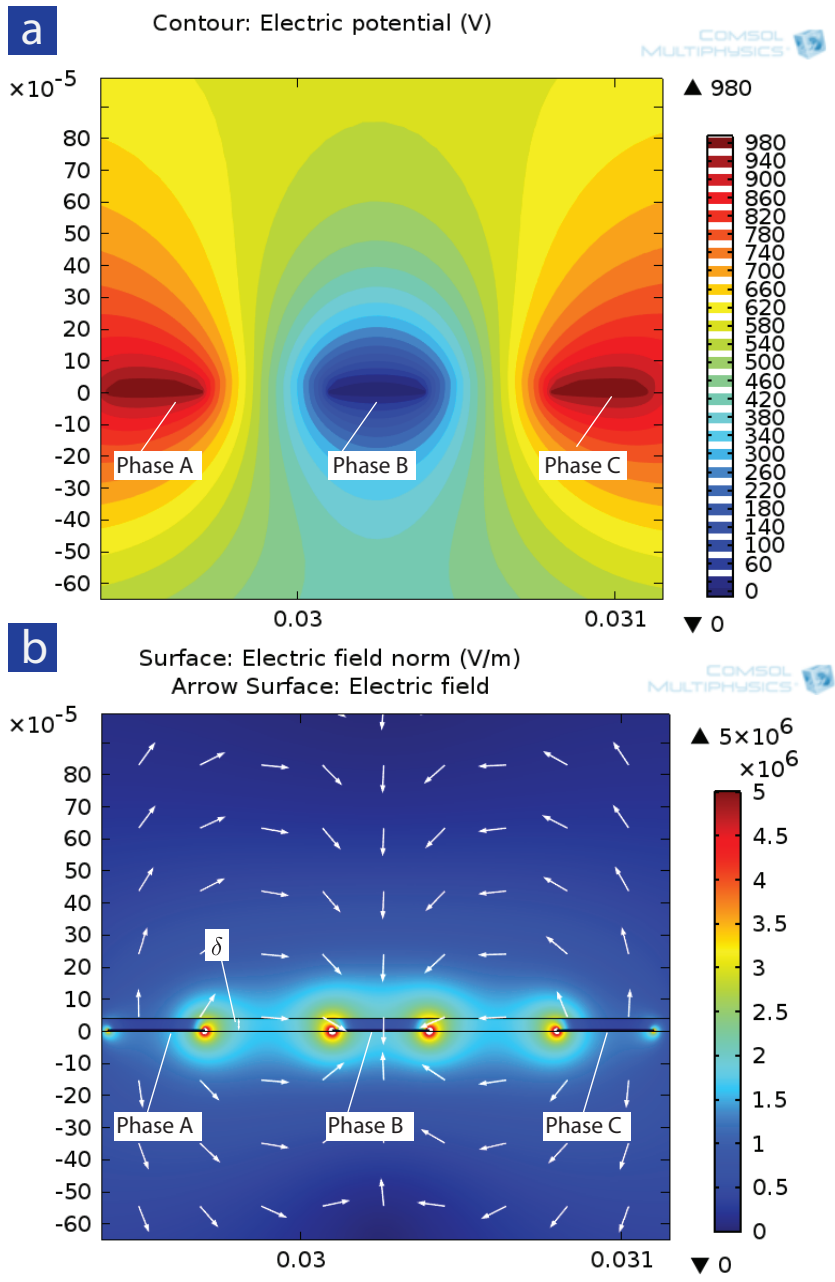

Figure 4: Numerical simulation results using finite element analysis software for the (a) electric potential (unit: V) and (b) electric field norm $|\mathbf{E}|$ (unit: V/m) and electric vector field in one fundamental spatial period for the EDS model. The units of the vertical and horizontal axes are in meters. The height of the electrodes have been considered to be $5 \mu \mathrm{m}$. The voltages of phases $\mathrm{A}, \mathrm{B}$, and $\mathrm{C}$ with are $1 \mathrm{kV}, 0$, and $1 \mathrm{kV}$, respectively. The electrode width, inter-electrode spacing, and thickness of the dielectric coating have been considered to be $300 \mu \mathrm{m}, 400 \mu \mathrm{m}$, and $50 \mu \mathrm{m}$, respectively. The relative permittivity of the dielectric coating is $\epsilon_{d}=3$.

examine the accuracy of the analytical solutions, we have defined the parameter "error percentage" as:

$$
\text { Error Percentage }=\frac{\mid \text { FEA }- \text { ANA } \mid}{\text { FEA }} \times 100 \% \text {. }
$$

The maximum value of error percentage in one fundamental spatial period in Fig. 5 is 1.6\%, 3.6\%, $2.9 \%, 5.9 \%, 6.7 \%, 5.5 \%$ for cases 1 through 6 , respectively. The most significant discrepancy between the two methods in Fig. 5 occurs when adjacent electrodes are both at zero potential or both at $1 \mathrm{kV}$. The difference between the results from the two methods is attributable to the fact that the voltage change between adjacent electrodes has been considered to be linear in the analytical approach, while the FEA 
method provides a more accurate voltage profile.

For instance, in Fig. 5 case 2, the analytical method assumes zero electric potential along the $x$ axis between the second and third electrodes, both having zero potential. However, the voltage profile provided by the FEA method increases from 0 in the second electrode to $31.1 \mathrm{~V}$ and then decreases to zero again when it reaches the third electrode. On similar lines, in Fig. 5 case3, when the first and second electrode are at $1 \mathrm{kV}$ electric potential, the voltage profile provided by the FEA method shows a decrease in electric potential between the aforementioned electrodes down to $957.2 \mathrm{~V}$ while the voltage is assumed to be still at $1 \mathrm{kV}$ along the $x$-axis in ANA method. Analytical models have been derived for the electric potential between two cylindrical conductors, like [32] or two-wire transmission line [33], that can be potentially used to provide better approximations for the voltage profile closer to what FEA method provides, rather than a linear one. Providing a detailed model of the voltage profile will improve the accuracy the analytical method, which can be a promising research topic for further studies.

\subsection{Impact of Dielectric Thickness}

As provided in the Eq. 5, the electric field distribution in the air is explicitly dependent upon the thickness of the dielectric layer $\delta$. Figure 6 depicts how the normalized maximum of $|\mathbf{E}|, E_{y}$, and $E_{x}$ on the EDS surface in one fundamental spatial period are dependent upon the thickness of the dielectric coating. The normalization is Fig. 6 is performed with respect to electric field distribution at $y=0$ when there is no dielectric coating: the electrodes are exposed directly to air. Although when the dielectric thickness increases in Fig. 6, the normalized maximum in the three curves decreases significantly, the drop in the $E_{y}$ is more significant, followed by $|\mathbf{E}|$ and $E_{x}$. As $\delta$ increases from 0 to $200 \mu \mathrm{m},|\mathbf{E}|, E_{x}$, and $E_{y}$ decrease up to approximately $54 \%, 30 \%$, and $68 \%$, respectively. When dielectric thickness increases from 0 to $50 \mu \mathrm{m}$, the $|\mathrm{E}|, E_{x}$, and $E_{y}$ experience losses about $25 \%, 12 \%$, and $38.7 \%$, respectively. This decrease is less prominent for higher values of $\delta$. For instance, when $\delta$ increases from $100 \mu \mathrm{m}$ to 150 $\mu \mathrm{m}$, the corresponding losses in $|\mathbf{E}|, E_{x}$, and $E_{y}$ are $15.5 \%, 7.4 \%$, and $17.8 \%$, respectively.

\subsection{Impact of Relative Permittivity}

Figure 7 (a) shows the normalized maximum values of the $|E|$ on the EDS surface in one fundamental spatial period as a function of relative permittivity. The normalization is done with respect to $\epsilon_{d}=1$ : dielectric layer is air. As can be observed in Fig. 7, the normalized values of the $|\mathbf{E}|$ increases as the dielectric permittivity of the dielectric coating increases. The slope of the curve is high at lower values 
(1)

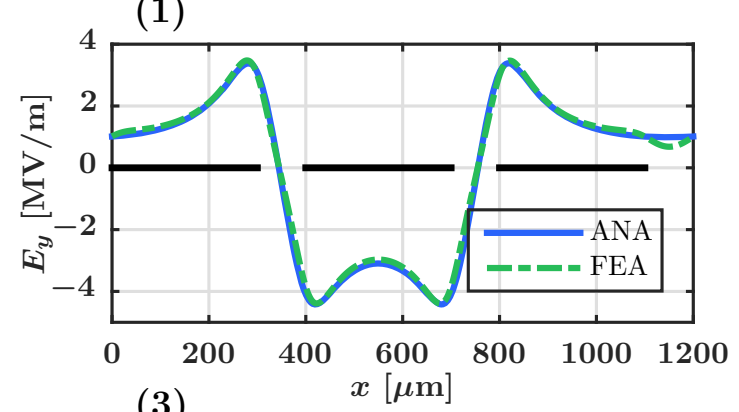

(3)

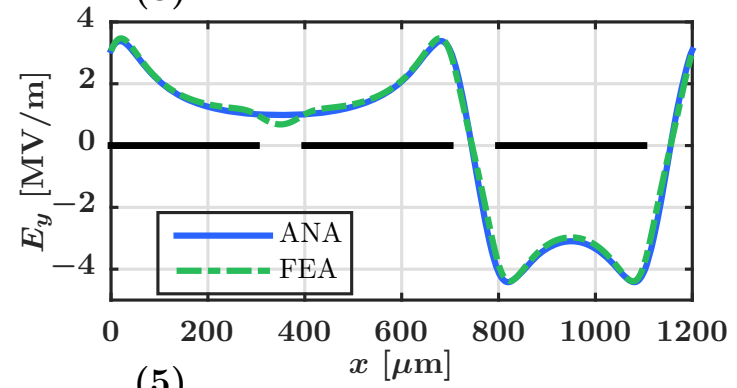

(5)

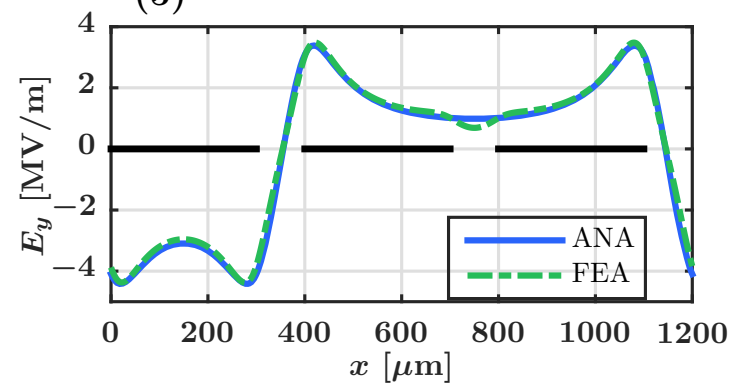

(2)

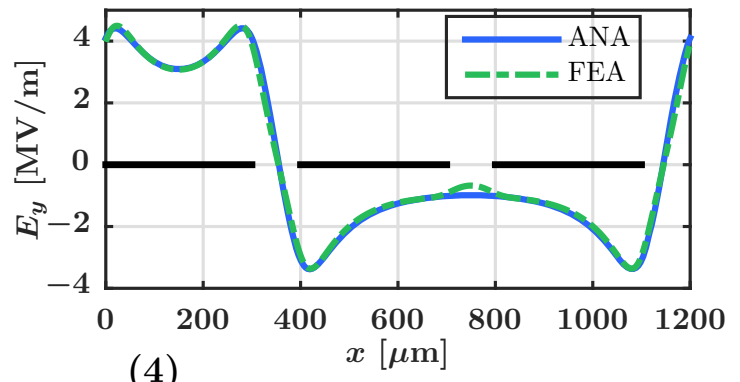

(4)

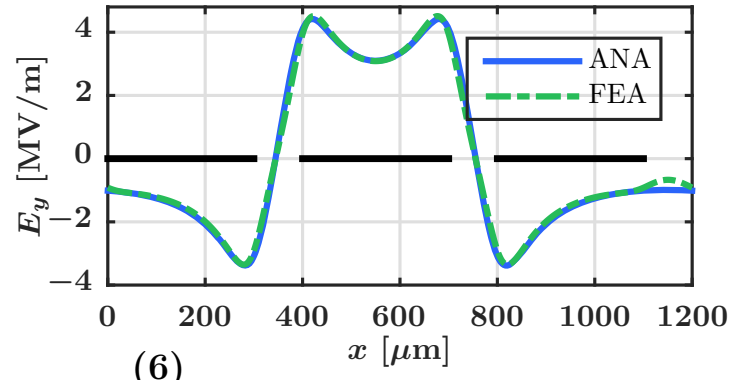

(6)

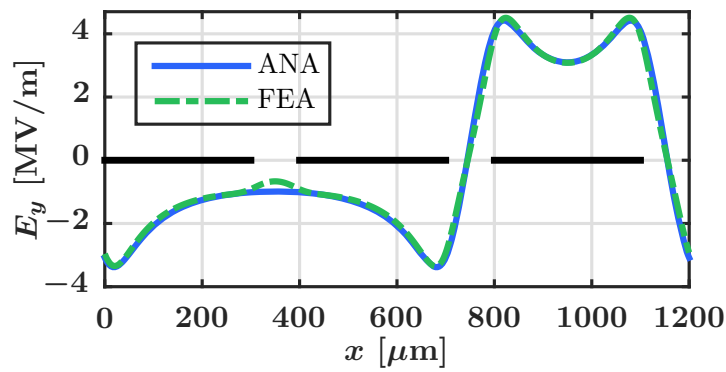

Figure 5: Comparison of analytical (ANA) results with numerical simulations using finite element analysis (FEA) software for $w=300 \mu \mathrm{m}$ and $g=100 \mu \mathrm{m}$ in one fundamental spatial period $T_{s}=1200 \mu \mathrm{m}$ for six different voltage combinations. The normal component of the electric field (unit: MV/m) on the EDS surface is illustrated vs. $x$. The positions of the first, second, and third electrodes are at $[0,300 \mu \mathrm{m}],[400 \mu \mathrm{m}, 700 \mu \mathrm{m}]$, and [800 $\mu \mathrm{m}, 1100 \mu \mathrm{m}]$, respectively. The amplitude of the voltage pulses is set at $1 \mathrm{kV}$. The thickness of the dielectric coating is $50 \mu \mathrm{m}$ and its relative permittivity is 3 .

of the relative permittivity while it decreases very quickly after it passes the relative permittivity of 10 . Five different candidate dielectric materials used for the EDS, namely Borosilicate Glass, Polyurethane (PU), Ethylene Tetrafluoroethylene (ETFE), Polyethylene Terephthalat (PET), and a thin film layer are highlighted in Fig. 7(b). As Fig. 7(b) indicates, using ETFE, PET, PU, Borosilicate Glass, and thin film layer give rise to $19 \%, 22 \%, 24 \%, 27 \%$, and $29 \%$ increase in the normalized of the maximum of electric field intensity, respectively, compared to air. Although Fig. 7(a) shows the behavior of the maximum of the $|\mathbf{E}|$ as a function of relative permittivity, the maximum of tangential and normal components of the electric field distribution have similar behaviors.

The behavior observed in Fig. 7(a) is justifiable using the capacitance concept. The two adjacent electrodes in the EDS configuration of Fig. 1 make a capacitor in which the dielectric medium of the 


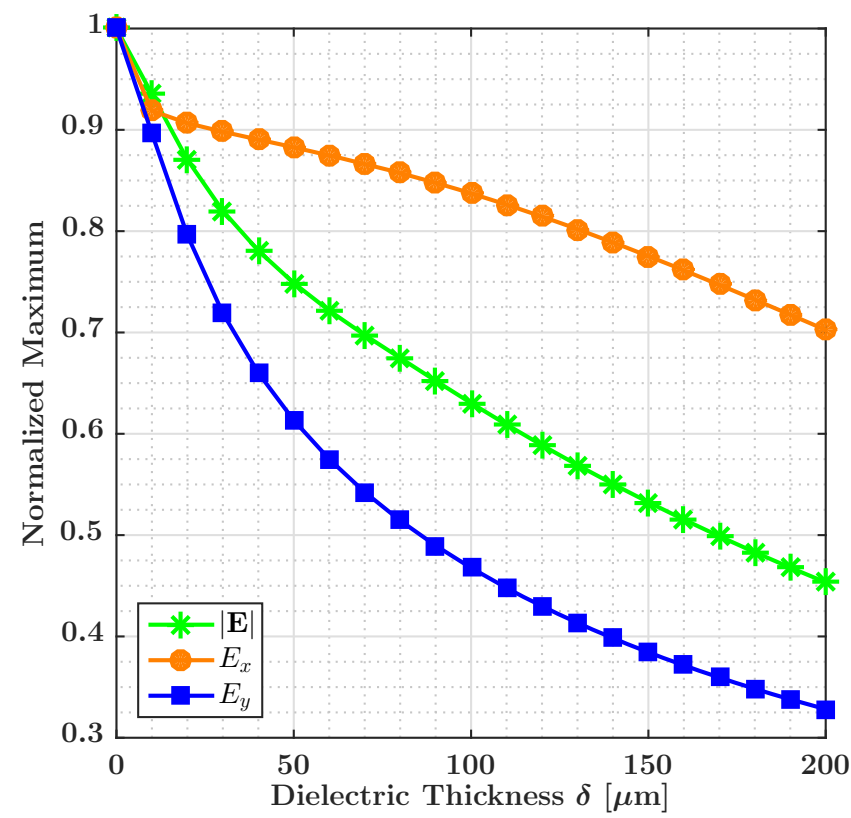

Figure 6: The impact of the thickness of the dielectric layer $\delta$ in the normalized maximum of electric field components and E. The electrode width and inter-electrode spacing are set to be $100 \mu \mathrm{m}$ and $700 \mu \mathrm{m}$, respectively. The relative permittivity of the dielectric layer is $\epsilon_{d}=3$. The voltages of the phases $\mathrm{A}, \mathrm{B}$, and $\mathrm{C}$ are set to be $1 \mathrm{kV}, 0$, and $1 \mathrm{kV}$, respectively.

equivalent capacitor consists of the transparent dielectric layer and air. Since the dielectric constant is a measure of how the material concentrates electric flux, increasing relative permittivity of the transparent dielectric layer will increase the electric field intensity on the EDS surface.

\subsection{Impacts of Inter-electrode Spacing and Electrode Width}

As denoted in Section 2, the electric field distribution on the EDS surface is dependent upon the electrode width and inter-electrode spacing through the Fourier coefficient $a_{k}$ and $b_{k}$ and subsequently $g_{k}$ and $h_{k}$ and $\Omega_{0}=\frac{2 \pi}{3(w+g)}$ in Eq. 5. The impacts of the aforementioned parameters on the maximum of the electric field magnitude $|\mathbf{E}|$ on the EDS surface in one fundamental spatial period have been shown in Fig. 8. As can be seen in Fig. 8(a), as the electrode width increases, the maximum of $|\mathbf{E}|$ decreases for a specific value of $g$; this decrease is more significant for smaller values of $w$. For instance, when $g=600 \mu \mathrm{m}$ and $w$ changes from 50 to 100,100 to 150 , and then 150 to $200 \mu \mathrm{m}$, the percent of decrease is $9 \%, 6 \%$, and $4 \%$, respectively. Figure 8 (b) provides the maximum of $|\mathbf{E}|$ as a function of $w$ for four values of $g$. The effect of change in the $g$ is more significant between trends in Fig. 8(b). When $w=100$ $\mu \mathrm{m}$ and $g$ changes from 100 to 200,200 to 300 , and then 300 to $400 \mu \mathrm{m}$, the percentages of decrease are $25.8 \%, 19.5 \%$, and $15.1 \%$, respectively.

In the design of the electrode geometry for EDS film, the two most important ones are (1) high optical 
(a)

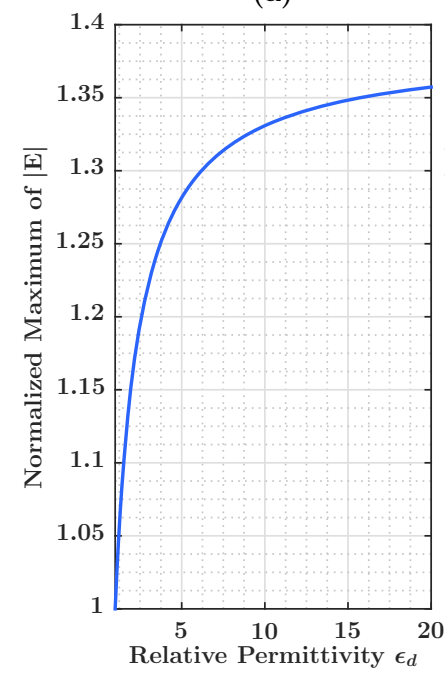

(b)

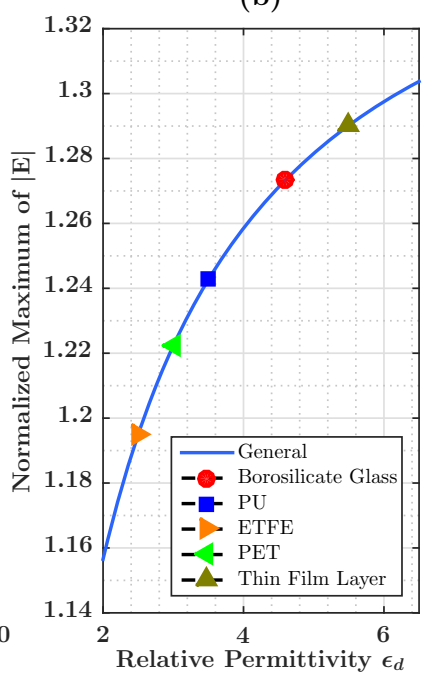

Figure 7: Normalized maximum of $|\mathbf{E}|$ as a function of relative permittivity $\epsilon_{d}$ (a) and the magnified part of the curve (b). The thickness of the dielectric coating has been considered $100 \mu \mathrm{m}$. The voltages of the phase $\mathrm{A}, \mathrm{B}$, and $\mathrm{C}$ are set to be $1 \mathrm{kV}$, 0 , and 0 , respectively. The electrode width and inter-electrode spacing are $100 \mu \mathrm{m}$ and $700 \mu \mathrm{m}$, respectively. The relative permittivities for Borosilicate Glass, PU, ETFE, PET, and thin film layer are 4.6, 3.5, 2.5, 3, and 5.5, respectively.

transmittance of the film and of the electrodes and (2) high dust removal efficiency (DRE) of the film to provide a traveling electric field at the EDS surface with sufficient strength for charging and repelling the dust particles by Coulomb force, and thus removing the dust layer from the surface. These are conflicting requirements in that high transparency of EDS film requires the electrode width $w$ to be as small as possible and the inter-electrode spacing $g$ to be as large as possible. For instance, if the electrodes occupy only $10 \%$ of the area of the EDS and the electrodes are $90 \%$ transparent, there will be only $1 \%$ transmission loss caused by the lamination of the EDS film over a solar module, e.g. a photovoltaic cell. However, for efficient charging of the dust particles imparted by the electrode activation by high voltage pulses, the electric field intensity needs to have sufficient strength across the entire surface of the EDS film for removing the dust layer by Coulomb force without reaching dielectric breakdown field causing corona discharge. If $w$ is too small, there will be a local intense electric field surrounding the electrodes embedded between the dielectric layer at the expense of lowering the $E_{y}$ component of the field over the surface in the gap regions between the adjacent electrodes. If $g$ is too large, the dust removal efficiency decreases particularly for small particles. It should be emphasized that that both electrostatic and optical characteristics of the EDS are important in the EDS design procedure. However, this study deals only with the electrostatic performance of the EDS in dust removal process. 
(a)

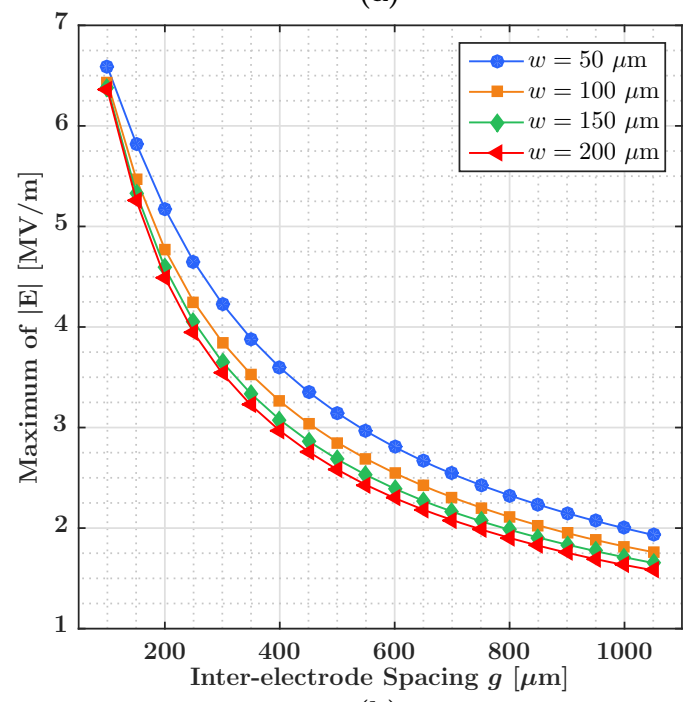

(b)

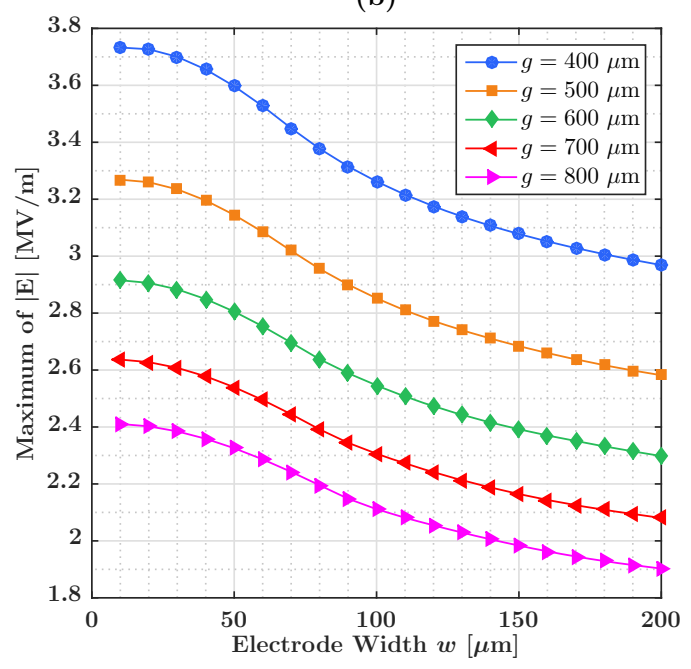

Figure 8: The impacts of (a) inter-electrode spacing $g$ and (b) electrode width $w$ on the maximum of electric field norm (unit: $\mathrm{MV} / \mathrm{m}$ ) on the EDS surface in one fundamental spatial period. The voltages of phases $\mathrm{A}, \mathrm{B}$, and $\mathrm{C}$ are set to be $1 \mathrm{kV}, 0$, and $1 \mathrm{kV}$, respectively. The thickness and relative permittivity of the dielectric coating are $50 \mu \mathrm{m}$ and 3 , respectively.

\section{Discussion on the multipolar DEP forces}

The closed-form expressions for the calculation of DEP forces up to the third-order were obtained in Section 2. In this section, we consider the numerical implementation of the mulipolar DEP forces and investigate the influential parameters. Although the DEP forces are calculated up to the third-order in Section 2, we have reported on the first-order DEP force in this section. Considering the numerical values of the design parameters used in current EDS prototypes, the second-order and third-order DEP forces are smaller than the first-order DEP forces by couple of order of magnitudes. Therefore, they have not been discussed in the numerical implementation. 
Figure 9 shows the first-order DEP forces exerted on a particle with $5 \mu \mathrm{m}$ radius in one fundamental spatial period for cases of 1,2, and 3 of the voltage combinations, as highlighted in Fig. 3. The DEP forces for the cases 4, 5, and 6 are identical to 1,2, and 3, respectively. The illustrated DEP forces for the three cases are identical except a shift of $w+g=\frac{T_{s}}{3}$ along the $x$ axis. Since $F_{y}^{(1)}$ is negative, i.e. it always points towards $-y$ or towards the EDS surface, we have considered the $\left|F_{y}^{(1)}\right|$ in Fig. 9. Negativity of the vertical component of the first-order DEP force $F_{y}^{(1)}$ is consistent with the fact that the dust particle experiences positive DEP force. As can be seen from Eq. 5, the magnitude of the $E_{y}$ monotonically decreases as $y$ increases. However, the magnitude of $E_{x}$ decreases and increases continually along the $x$ axis in one fundamental spatial period. Since the gradient of the electric field component determines the direction of the DEP force, the $F_{x}^{(1)}$ changes sign continuously in one fundamental spatial period while $F_{y}^{(1)}$ is always points towards the EDS surface.

In Fig. 9 the maximum and minimum values of the $F_{x}^{(1)}$ are $0.54 \mathrm{nN}$ and $-0.54 \mathrm{nN}$, respectively. The magnitude of the vertical component of the first-order DEP force reaches its maximum and minimum values of $1.25 \mathrm{nN}$ and $1.56 \mathrm{pN}$, respectively. The average value of the vertical component of the firstorder DEP force that the dust particle experiences along the $x$ axis in one fundamental spatial period is $0.21 \mathrm{nN}$. For comparison purposes, if we assume the density of the particle is $2300 \mathrm{~kg} / \mathrm{m}^{3}$, the weight of the particle is calculated as $11.8 \mathrm{pN}$.

Similar to the study on the effect of dielectric thickness on the electric field intensity on the EDS surface, we examine how dielectric film thickness $\delta$ affects the first-order DEP forces that a test dust particle experiences in one fundamental spatial period on the EDS surface. Figure 10 illustrates how the $\left|F_{y}^{(1)}\right|$ changes along the $x$ axis in one fundamental spatial period for different values of $\delta$. Since the electrode width and inter-electrode spacing are considered to be 100 and $700 \mu \mathrm{m}$, respectively, the first, second, and third electrodes are located at [0,100 $\mu \mathrm{m}],[800 \mu \mathrm{m}, 900 \mu \mathrm{m}]$, and [1600 $\mu \mathrm{m}, 1700 \mu \mathrm{m}]$, respectively, in Fig. 10. Noting the positions of the electrodes, it is clear that when the test dust particle sitting right on the top of the tips of the electrodes, it experiences the maximum DEP force, while when it moves along the $x$ axis and reaches the center of the electrodes, the DEP force continuously decreases. Once it travels from the center of the electrodes and reaches the end tip of the electrodes, it experiences an increasing DEP force. This behavior is clearly recognizable when $\delta=20 \mu \mathrm{m}$ and and to some extent when $\delta=40 \mu \mathrm{m}$. However, it gets less salient when $\delta$ increases and reaches $100 \mu \mathrm{m}$.

In Fig. 10, as $\delta$ increases, the amplitudes of the maxima and minima of the DEP force continuously 

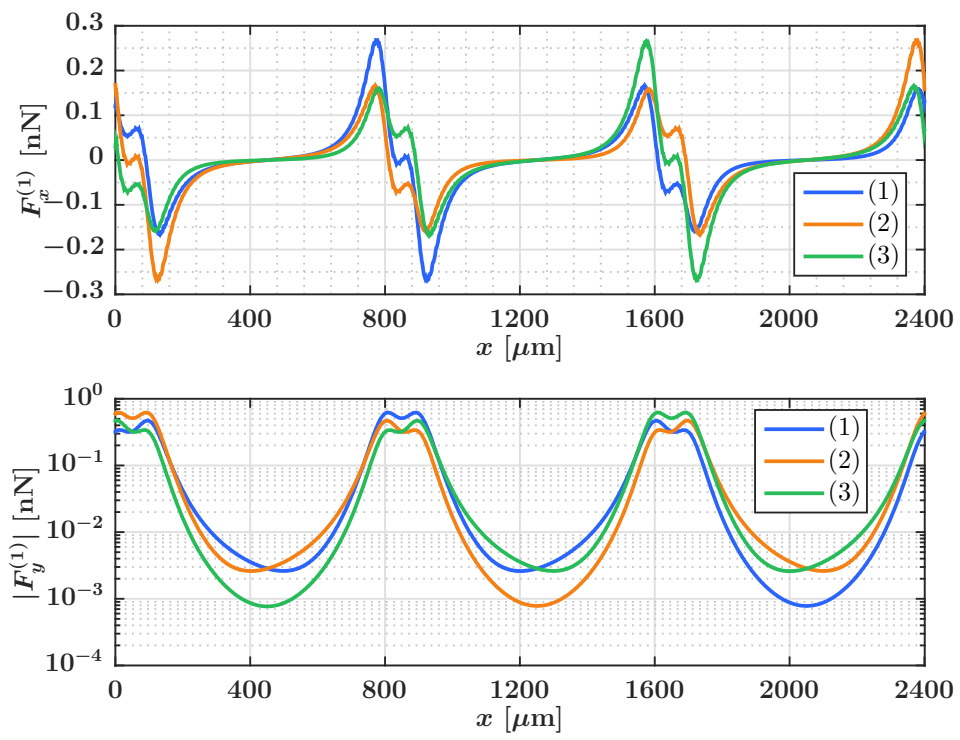

Figure 9: First-order DEP forces in $x$ - and $y$-direction: (top) $F_{x}^{(1)}$ (unit: $\mathrm{nN}$ ) and (bottom) $\left|F_{y}^{(1)}\right|$ (unit: $\mathrm{nN}$ ) for three cases of 1,2 , and 3 of the voltage combinations, as highlighted in Fig. 3 . The thickness of the dielectric coating and radius of the spherical lossless dust particle are 50 and $5 \mu \mathrm{m}$, respectively. The electrode width and inter-electrode spacing are 100 and $700 \mu \mathrm{m}$, respectively. The relative permittivity of the transparent dielectric layer and the particle are assumed to be 3 and 4 , respectively. The amplitude of voltage pulses is $1 \mathrm{kV}$.

decrease, but this decrease is more prominent when $\delta$ changes from $20 \mu \mathrm{m}$ to $40 \mu \mathrm{m}$ than when it changes from $80 \mu \mathrm{m}$ to $100 \mu \mathrm{m}$. For instance, $\left|F_{y}^{(1)}\right|$ experiences $53.7 \%$ decrease when $\delta$ changes from 20 to 40 $\mu \mathrm{m}$, while this decrease is $28 \%$ when $\delta$ changes from $80 \mu \mathrm{m}$ to $100 \mu \mathrm{m}$. The spikes in the curve for 100 $\mu \mathrm{m}$ is attributable to the fact that the $\left|F_{y}^{(1)}\right|$ is very close to 0 . In Fig. 10 , the test dust particle with $5 \mu \mathrm{m}$ radius experiences an average value of first-order DEP force of $0.47,0.26,0.17$, and $0.12 \mathrm{nN}$ for $\delta=20$, 40,60 , and $80 \mu \mathrm{m}$, respectively.

Figure 11 depicts the behavior of the vertical component of the first-order DEP force and Coulomb force as a function of particle radius for four different applied voltages. To calculate the Coulomb force in Fig. 11, we have assumed that the dust particle is charged positively to its saturation charge level of $q=4 \pi R^{2} \sigma_{s}$, in which $R$ is the particle radius and $\sigma_{s}=27 \mu \mathrm{C} / \mathrm{m}^{2}$ [34]. It is noted that in Fig. 11, the direction of the vertical component of the first-order DEP force is towards $-y$ while the Coulomb force points towards $+y$. When the applied voltage is $1,1.2,1.4$, and $2 \mathrm{kV}$, the maximum of the electric field magnitude reaches 2.26, 2.7, 3.16, and $4.5 \mathrm{MV} / \mathrm{m}$, respectively, on the EDS surface. When the applied voltage changes from $1 \mathrm{kV}$ to $2 \mathrm{kV}$, the Coulomb force shows $100 \%$ increase, which is completely expected as the electric field intensity follows the voltage pattern in a linear fashion, while the DEP force has an $300 \%$ increase. 


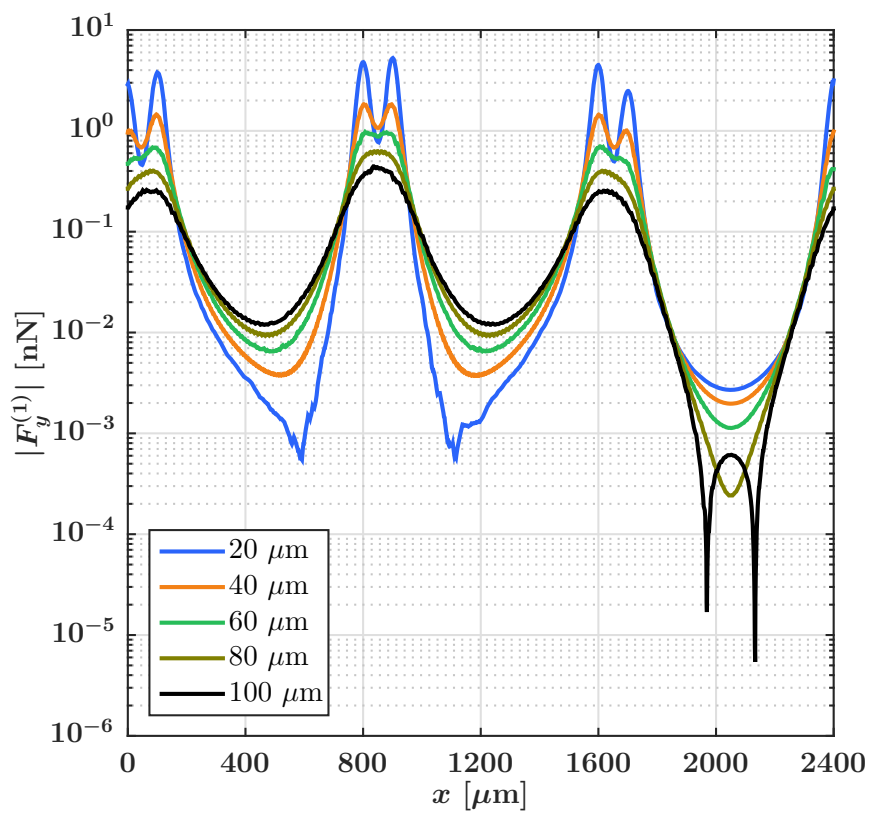

Figure 10: The impact of the thickness of transparent dielectric material on the magnitude of the vertical component of the first-order DEP force (unit: $\mathrm{nN}$ ) in one fundamental spatial period in one fundamental spatial period, exerted on a resting dust particle with $5 \mu \mathrm{m}$ radius. The electrode width and inter-electrode spacing are 100 and $700 \mu \mathrm{m}$, respectively, and the voltages of first, second, and third electrodes are $1 \mathrm{kV}, 0$, and $1 \mathrm{kV}$, respectively.

\section{Force Comparison}

Since the ultimate goal of using EDS is removing dust particles from the optical surfaces of solar collectors, having a comparison between the adhesion forces and repelling forces is of utmost importance in order to evaluate the EDS performance. To perform this comparison, we consider Fig. 12(a), in which the particle is sitting right on top of the energized electrode, where it experiences the maximum of the first-order DEP force in the $-y$ direction, as addressed in Fig. 9. Since the electrode is energized, the electric field distribution right above the electrode on the EDS surface experiences the maximum of the $E_{y}$ in the $+y$ direction. The free body diagram of the vertical forces exerted on the particle is illustrated in Fig. 12(b). It is assumed that the particle is positively charged and all the depicted forces are exerted on the center of the particle. Exerted forces addressed in Fig. 12 are calculated as follows:

1. Gravity $F_{g}$ : the gravitational force for a spherical particle with radius $R$ is calculated as

$$
F_{\mathrm{g}}=\frac{4}{3} \pi \rho R^{3} g
$$

in which $\rho$ is the density of the particle and $g$ is the gravitational acceleration. 


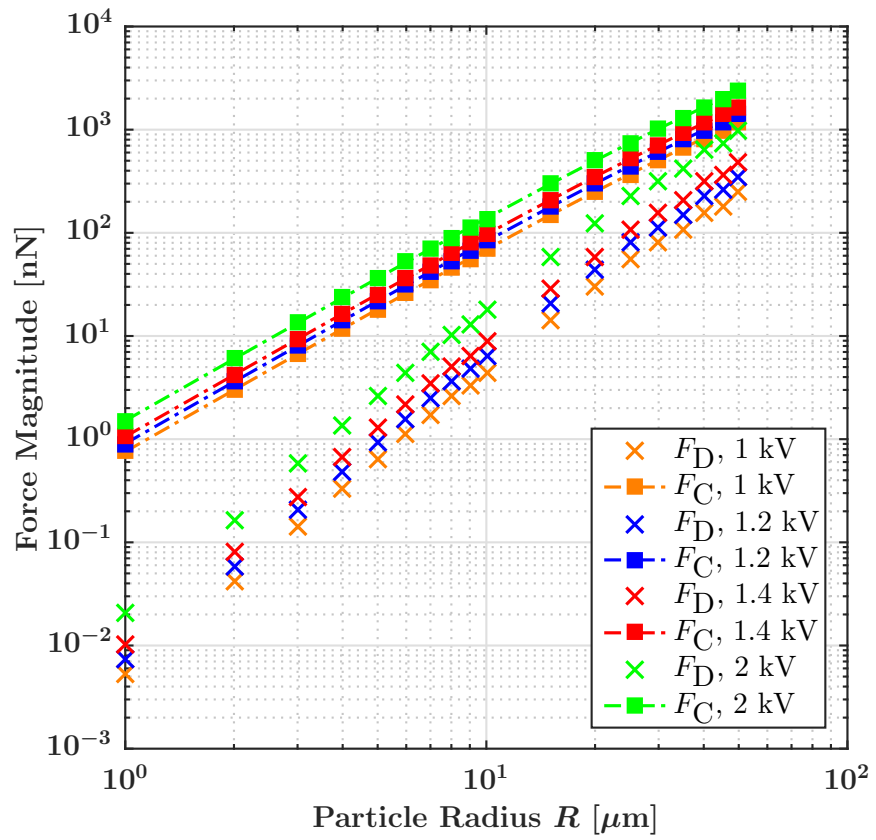

Figure 11: Variation of the magnitude of vertical component of the first-order DEP force $\left|F_{y}^{(1)}\right|$ and Coulomb force as a function of particle radius for four different applied voltages. The electrode width and inter-electrode spacing are $100 \mu \mathrm{m}$ and $700 \mu \mathrm{m}$, respectively. The thickness of the dielectric coating is $50 \mu \mathrm{m}$ and its relative permittivity is 3 . The electric potential of electrodes A, B, and $\mathrm{C}$ are $V, 0$, and $V$, respectively, in which $V$ is the applied voltage. The test particle is positioned right on top of the energized electrode $\mathrm{C}$.

2. Coulomb Force $F_{\mathrm{C}}$ : the vertical component of the Coulomb force exerted on the particle is obtained from the expression $F_{\mathrm{C}}=q E_{y}$, in which $E_{y}$ is the vertical component of the electric field distrbution calculated in the center of the particle and $q$ is the electric charge of the particle. We assume the particle is positively charged to its saturation level. The electric charge of the particle is obtained from the following expression:

$$
q=4 \pi R^{2} \sigma_{s}
$$

in which $\sigma_{s}$ is the surface saturation charge of the particle and $R$ is the particle radius.

3. Image force $F_{\mathrm{i}}$ : the image force is calculated from the following expression:

$$
F_{\mathrm{i}}=\frac{1}{4 \pi \epsilon_{0} \epsilon_{d}} \frac{q^{2}}{[2(\delta+R)]^{2}},
$$

in which $\epsilon_{d}$ is the relative permittivity of the dielectric layer, $\delta$ is the thickness of the dielectric layer, and $q$ is the charge of the particle obtained from Eq. 40. It should be noted that this expression is valid if the size of the particle is much smaller than the electrode width. If the 
particle size is comparable with the electrode width, Eq. 41 is merely an approximation to the exact image force.

4. Adhesion force $F_{\mathrm{a}}$ : the adhesion force is considered to be the summation of the two forces when the particle is in contact with the surface: van der Waals force and capillary force, denoted as $F_{\mathrm{vdW}}$ and $F_{\text {cap }}$, respectively. The mathematical expression for the van der Waals force between perfectly smooth particle and surface with no roughness is calculated from the following expression [35]:

$$
F_{\mathrm{vdW}}=\frac{A_{h} R}{6 D^{2}}
$$

in which $R$ is the radius of the adhering particle, $D \approx 0.3-0.4 \mathrm{~nm}$ is the equilibrium separation between two solid surfaces, and $A_{h}$ is the Hamaker constant. Typical values for the Hamaker constant is in the order of $10^{-19} \mathrm{~J}$ in vacuum. The capillary force $F_{\text {cap }}$, as depicted in Fig. 12 (c), is obtained from the following formula [36]:

$$
F_{\text {cap }}=2 \pi R\left[\cos \left(\theta_{1}\right)+\cos \left(\theta_{2}\right)\right] \gamma_{W},
$$

in which $\theta_{1}$ and $\theta_{2}$ are the contact angles of particle and surface, respectively, and $\gamma_{W}$ the surface tension of water. It should be noted that Eq. 43 is merely an approximation for the capillary force which is independent of the relative humidity. As has been shown in different experimental studies such as [37], the capillary force increases significantly with relative humidity. However, for the sake of initial comparison of forces, we use the simplified expression of capillary force provided in Eq. 43 .

Figure 13 illustrates the behavior of different forces exerted on the particle as a function of particle radius $R$. The numerical parameters used to calculate the forces in Fig. 13 are provided in Table 1 . As Fig. 13 depicts, the capillary force is the predominant adhering force, followed by the van der Waals force. As the van der Waals force is smaller than the capillary force by an order of magnitude, the summation of these forces, the so-called adhesion force, is very close to the capillary force. The Coulomb force acting as the repelling force is approximately three orders of magnitude smaller than the adhesion force. When $\delta$ increases, image force, DEP force, and Coulomb force all decrease, as addressed previously, while gravity, capillary force, and van der Waals force do not change, assuming the surface roughness do not change with the increase of dielectric thickness. 

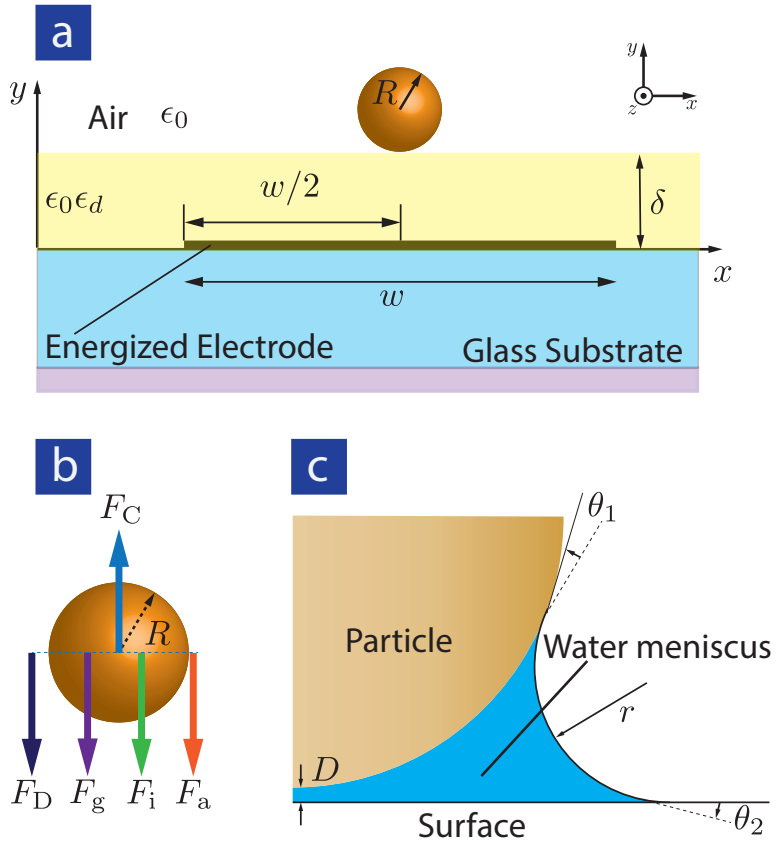

Figure 12: (a) dust particle with radius $R$ sitting right above the energized electrode, (b) free-body diagram of the forces exerted on the particle in the $y$-direction. The terms $F_{\mathrm{D}}, F_{\mathrm{g}}, F_{\mathrm{i}}, F_{\mathrm{a}}$, and $F_{\mathrm{C}}$ denote the vertical component of the first-order DEP force, gravity, image force, adhesion force, and vertical component of the Coulomb force, respectively, (c) formation of water meniscus between the particle and the representation of the capillary force. $\theta_{1}$ and $\theta_{2}$ are the particle and surface contact angles, respectively, and $D$ denotes the equilibrium distance between two solids and is $\approx 0.3-0.4 \mathrm{~nm}$. The equilibrium radius of the meniscus is denoted as $r$.

Based upon what Fig. 13 suggests, the EDS is not able to remove any particle size with the current design even when the particle acquire maximum possible surface charge and maximum vertical electric field component. In other words, the adhering force is much stronger than the repelling force. However, the model for the van der Waals force and capillary force considered in Fig. 13 are the ideal models, in which a perfectly smooth spherical particle is in contact with a smooth surface which none of them do not possess any asperities. In practice, both particle and surface possess asperities that affect the ideal models expressed in Eqs. 42 and 43 significantly. This is a point that has received significant attention by different researchers working in the field of particle surface retention and resuspension. As highlighted by Rabinovich et al. [38], presence of roughness of about 1 or $2 \mathrm{~nm}$ root mean square (RMS) in the surface decreases the adhesion forces calculated for an ideal model by an order of magnitude or more.

In order to have a more thorough and realistic idea of the magnitude of the adhesion forces exerted on the particle, we consider a commercially available thin film layer, a promising candidate as the top transparent dielectric layer of the EDS, in which its surface roughness is known. According to its fact sheet, the roughness average denoted as $R_{a}$ and peak to valley roughness, denoted as $R_{p v}$, are $<0.5 \mathrm{~nm}$ 


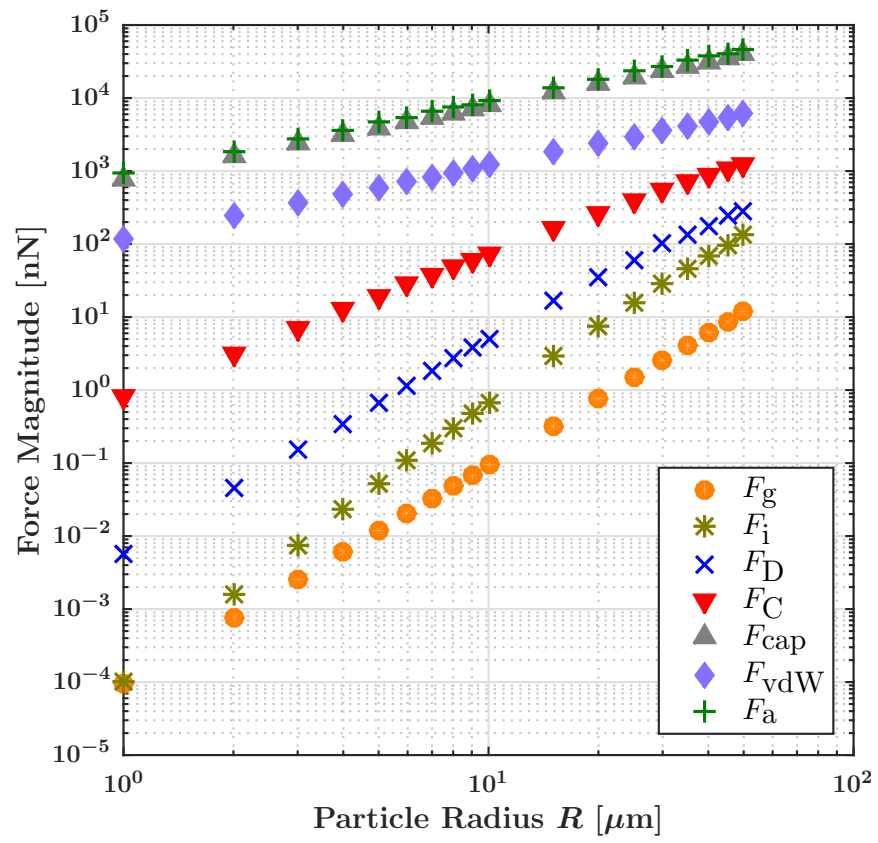

Figure 13: Comparison of the attracting and repelling forces exerted on the particle as a function of particle radius when the particle is sitting right on top of the energized electrode. The electrode width and inter-electrode spacing are 100 and 700 $\mu \mathrm{m}$, respectively, and the electrode has $1 \mathrm{kV}$ electric potential.

and $<20 \mathrm{~nm}$, respectively. It is noted that the $R_{a}$ is the arithmetic average of the absolute values of the profile height deviations from the mean line, recorded within the evaluation length. The root mean square of the surface roughness, denoted as RMS, is defined as the average of the profile height deviations from the mean line, recorded within the evaluation length. Assuming the profile height function has a sinusoidal pattern with a particular spatial period along the horizontal axis, the relationship between the $R_{a}$ and RMS values is obtained as follows:

$$
\mathrm{RMS}=1.1 R_{a}
$$

Hence, for the thin film transparent layer, the RMS $<0.55 \mathrm{~nm}$.

According to the model developed by Rabinovich et al. [38], the van der Waals force between a particle and a surface with nanoscale roughness, based upon our notations and symbols in this paper, is expressed as

$$
F_{\mathrm{vdW}}=\frac{A_{h} R}{6 D^{2}}\left[\frac{R_{p v}^{2}}{R_{p v}^{2}+63.95 R R_{a}}+\frac{D^{2}}{\left(D+2 R_{a}\right)^{2}}\right],
$$

in which $A_{h}$ is the Hamaker constant of the surface, $R$ is the radius of the particle, and $D \approx 0.3 \mathrm{~nm}$. 


\begin{tabular}{lll}
\hline Variable & Desription & Numerical Value \\
\hline \hline $\mathrm{g}$ & gravitational acceleration & $9.8 \mathrm{~m} / \mathrm{s}^{2}$ \\
$\rho$ & density of the particle & $2300 \mathrm{~kg} / \mathrm{m}^{3}$ \\
$\epsilon_{d}$ & relative permittivity of the dielectric layer & 3 \\
$\delta$ & thickness of the dielectric layer & $40 \mu \mathrm{m}$ \\
$A_{h}$ & Hamaker constant & $6.5 \times 10^{-20} \mathrm{~J}$ \\
$D$ & equilibrium distance between two solid surfaces & $0.3 \mathrm{~nm}$ \\
$\gamma_{W}$ & surface tension of water at room temperature & $0.073 \mathrm{~N} / \mathrm{m}$ \\
$\theta_{1}, \theta_{2}$ & contact angle of particle/surface & $\pi / 6 \mathrm{rad}$ \\
$\epsilon_{p}$ & relative permittivity of the particle & 4 \\
$\sigma_{s}$ & surface saturation charge of the particle & $27 \mu \mathrm{C} / \mathrm{m}^{2}$ \\
\hline
\end{tabular}

Table 1: List of parameters used in the comparison of the forces

$\{$ You2013⿻1 1 392

The Hamaker constant of a particular material is related to its surface energy via the following equation [39]:

$$
A_{h}=1.44 \times 10^{-18} \gamma
$$

in which $\gamma$ is the surface energy of the material in $\mathrm{J} / \mathrm{m}^{2}$. The surface energy of the borosilicate glass is considered to be $0.04 \mathrm{~J} / \mathrm{m}^{2}$. Therefore, its Hamaker constant is $A_{h}=5.76 \times 10^{-20} \mathrm{~J}$.

The proposed model for the capillary force, when the surface roughness of the surface is taken into account, is expressed as [38]:

$$
F_{c}=4 \pi \gamma_{W} R \cos \theta\left[1-\frac{R_{a}}{r \cos \theta}\right]
$$

in which $\cos \theta=\left(\cos \theta_{1}+\cos \theta_{2}\right) / 2$ and $r$ is the equilibrium radius of the meniscus, as illustrated in Fig. 12(c). The radius of the meniscus is obtained from the following expression

$$
r=-\frac{\gamma_{W} V}{N_{a} k T \ln \left(p / p_{s}\right)}
$$

in which $V=18 \times 10^{-6} \mathrm{~m}^{3} / \mathrm{mol}$ is the molar volume of water, $\gamma_{W}=0.073 \mathrm{~N} / \mathrm{m}$ is the surface tension of water at room temperature, $N_{a}$ is the Avogadro constant, $k$ is the Boltzmann constant, $T$ is the temperature in Kelvin, and $p / p_{s}$ is the $\mathrm{RH}$ with $p$ the vapor pressure and $p_{s}$ the saturated vapor pressure. 
At room temperature, Eq. 48 is expressed as

$$
r=-\frac{5.27 \times 10^{-10}}{\ln \left(p / p_{s}\right)}
$$

The criteria for the meniscus formation between the two surfaces is the term inside the bracket in Eq. 47 should be positive, i.e.:

$$
R_{a} \leq r \cos \theta
$$

Assuming $\theta_{1}=\theta_{2}=\pi / 6$, the condition for meniscus formation and consequently presence of capillary force is $\mathrm{RH} \geq 41 \%$. For relative humidity values less than $40 \%$, there is no meniscus between the particle and the surface and consequently no capillary force.

Figure 14 provides a comparison between the vertical component of Coulomb force, identical to the one in Fig. 13, the modified van der Waals force, and capillary force for different values of RH. In Fig. 14, the van der Waals force drops more than 1 order of magnitude, compared to the ideal case, when the surface roughness is considered and calculated via Eq. 45. Although the Coulomb force starts from 0.93 and 1.35 order of magnitude lower than the curves for modified van der Waals force and capillary force for $\mathrm{RH}=41 \%$, respectively, it exceeds them after $R=8 \mu \mathrm{m}$ and $R=30 \mu \mathrm{m}$, respectively. In Fig. 14, as RH increases from $41 \%$ to $70 \%$, the capillary force increases and approaches the capillary force curve, calculated from Eq. 43, in which it is independent of RH. The increase of capillary force magnitude is much more prominent when $\mathrm{RH}$ changes from $41 \%$ to $50 \%$ rather than $50 \%$ to $60 \%$ or $60 \%$ to $70 \%$. The capillary force increases more than 1 order of magnitude when RH changes from $41 \%$ to $50 \%$ while the capillary force experiences approximately 0.1 order of magnitude increase when $\mathrm{RH}$ changes from $60 \%$ to $70 \%$.

\section{Conclusion}

We presented closed-form analytical solutions for the electrical potential and electrical field distribution in an electrodynamic screen (EDS) configuration with one layer of transparent dielectric coating. The impacts of different EDS design parameters including electrode width, inter-electrode spacing, thickness and relative permittivity of the transparent dielectric layer in the electric field distribution were addressed. It was shown that the vertical component of the electric field intensity on the EDS surface de- 


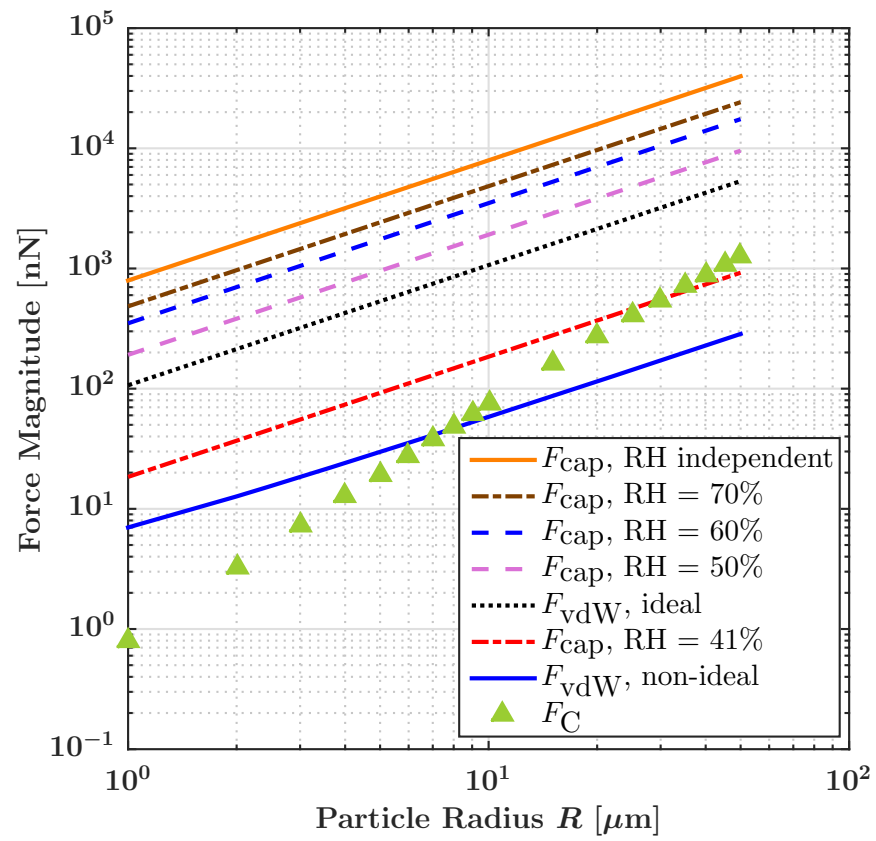

Figure 14: Comparison between the vertical component of Coulomb force and ideal (no surface roughness) and non-ideal (with surface roughness) van der Waals force and capillary force for several different relative humidity values. The values for the Coulomb force is identical to Fig. 13

creases significantly as the thickness of the dielectric coating increases. Based upon the analytical solutions of the electric field distributions, we provided the closed-form expressions for the dielectrophoretic (DEP) forces in the EDS configuration up to the third-order. Dependent upon the nonlinearity of the problem and EDS design parameters, the DEP forces expressions can be embedded efficiently in the equation of motion for particle trajectory modeling in further studies.

In evaluating the EDS performance in dust removal process, it is important that different attracting and repelling forces exerted on the dust particles compared with each other. Therefore, the electrostatic models proposed in the first part of this study and the mathematical models suggested for the other forces, in particular van der Waals force and capillary force, were compared. To have a more thorough and realistic comparison between the forces, we considered the surface roughness of the top EDS surface in the adhesion force model. The comparison results showed that capillary force dominates the van der Waals force by at least one order of magnitude when the relative humidity surpasses a threshold. Thus, it makes the Coulomb force ineffective in removing fine dust particles. Increase in relative humidity deteriorates the dust removal efficiency as the capillary force increases exponentially. This point corroborates the observation we had in experimental investigation of EDS performance in high relative humidity values previously. In this study, we included the surface roughness of the top EDS surface into the models to 
have a more realistic comparison between the attraction and repelling forces in the balance of forces exerted on dust particles. In further studies, the surface roughness of the particles can also be considered in the adhesion force models to make the comparisons more accurate and compliant with experimental observations. As we quantitatively addressed the detrimental impact of relative humidity increase, using superhydrophobic coatings for the top layer of the EDS is strongly recommended to maintain the dust removal efficiency in high relative humidity.

\section{Acknowledgement}

We acknowledge the grant from the Department of Energy (DOE CSP DE-EE0005794) for supporting a research project on prototype development and evaluation of self-cleaning concentrated solar power collectors.

\section{Nomenclature}

$\begin{array}{ll}\mathbf{a}_{x} & \text { Unit vector in } x \text { direction, } \mathrm{m} \\ \mathbf{a}_{y} & \text { Unit vector in } y \text { direction, } \mathrm{m} \\ a_{k}, b_{k} & \text { Fourier coefficients } \\ A_{h} & \text { Hamaker constant, } \mathrm{J} \\ c_{j} & \text { Cauchy product } \\ C_{i} & \text { Locally-defined constant } \\ D & \text { Equilibrium separation of two solids, } 0.3 \mathrm{~nm} \\ \mathbf{E} & \text { Electric field vector, } \mathrm{V} / \mathrm{m} \\ E_{x} & \text { Tangential component of the electric field, } \mathrm{V} / \mathrm{m} \\ E_{y} & \text { Normal component of the electric field, } \mathrm{V} / \mathrm{m} \\ \mathbf{F}^{n} & n^{\text {th }}-\text { order dielectrophoretic force, } \mathrm{N} \\ \mathbf{F}^{\text {tot }} & \text { Total dielectrophoretic force, } \mathrm{N} \\ F_{\mathrm{a}} & \text { Adhesion force, } \mathrm{N} \\ F_{\text {cap }} & \text { Capillary force, } \mathrm{N} \\ F_{\mathrm{C}} & \text { Coulomb force in } y \text {-direction, } \mathrm{N} \\ F_{\mathrm{i}} & \text { Image force in } y \text {-direction, } \mathrm{N} \\ F_{\mathrm{vdW}} & \text { van der Waals force, } \mathrm{N}\end{array}$




\begin{tabular}{|c|c|}
\hline$F_{x}^{(n)}$ & $n^{\text {th }}-$ order DEP force in $x$ direction \\
\hline$F_{y}^{(n)}$ & $n^{\text {th }}-$ order DEP force in $y$ direction \\
\hline$g$ & Inter-electrode spacing, $\mathrm{m}$ \\
\hline$g$ & Gravitational acceleration, $9.8 \mathrm{~m} / \mathrm{s}^{2}$ \\
\hline$g_{k}, h_{k}$ & Fourier coefficients \\
\hline i & Imaginary unit \\
\hline$K_{n}$ & Generalized polarization coefficient \\
\hline$k$ & Boltzman constant, $1.38 \times 10^{-23} \mathrm{~m}^{2} \mathrm{~kg} / \mathrm{s}^{2} \mathrm{~K}$ \\
\hline$n$ & Order of problem \\
\hline$N_{a}$ & Avogadro constant, $6.022 \times 10^{23} / \mathrm{mol}$ \\
\hline$p$ & Vapor pressure, $\mathrm{Pa}$ \\
\hline$p_{s}$ & Saturated vapor pressure, $\mathrm{Pa}$ \\
\hline$q$ & Dust particle charge, $\mathrm{C}$ \\
\hline$r$ & Equilibrium radius of the water meniscus, $\mathrm{m}$ \\
\hline$R$ & Dust particle radius, $\mathrm{m}$ \\
\hline$R_{a}$ & Surface roughness average, $\mathrm{m}$ \\
\hline$R_{p v}$ & Surface peak to valley roughness, $\mathrm{m}$ \\
\hline $\mathbf{S}^{(n)}$ & $n^{\text {th }}-$ order DEP force spatial factor \\
\hline$S_{x}^{n}$ & $n^{\text {th }}-$ order DEP force spatial factor in $x$ \\
\hline$S_{y}^{n}$ & $n^{\text {th }}$-order DEP force spatial factor in $y$ \\
\hline$t$ & Time, s \\
\hline$T$ & Temperature, $\mathrm{K}$ \\
\hline$T_{s}$ & Fundamental spatial period, m \\
\hline$T_{t}$ & Time period, s \\
\hline$V$ & Molar volume of water, $18 \times 10^{-6} \mathrm{~m}^{3} / \mathrm{mol}$ \\
\hline$V_{i}$ & Electric potential of phase $\mathrm{i}, \mathrm{V}$ \\
\hline$w$ & Electrode width, m \\
\hline$(x, y)$ & The Cartesian coordinates, $\mathrm{m}$ \\
\hline
\end{tabular}

\section{Greek Symbols}




$\begin{array}{ll}\gamma & \text { Surface energy of the material, } \mathrm{J} / \mathrm{m}^{2} \\ \gamma_{W} & \text { Surface tension of water, } 0.073 \mathrm{~N} / \mathrm{m} \\ \delta & \text { Thickness of the dielectric layer, } \mathrm{m} \\ \epsilon_{0} & \text { Permittivity of free space, } 8.854 \times 10^{-12} \mathrm{~F} / \mathrm{m} \\ \epsilon^{*} & \text { Complex dielectric permittivity, } \mathrm{F} / \mathrm{m} \\ \epsilon_{d} & \text { Relative permittivity of the dielectric layer } \\ \theta_{1} & \text { Contact angle of meniscus and particle, } \mathrm{rad} \\ \theta_{2} & \text { Contact angle of meniscus and surface, } \mathrm{rad} \\ \rho & \text { Density of the dust particle, } \mathrm{kg} / \mathrm{m}^{3} \\ \sigma & \text { Conductivity of the material, } \mathrm{S} / \mathrm{m} \\ \sigma_{s} & \text { Saturation charge, } 27 \mu \mathrm{C} / \mathrm{m}^{2} \\ \phi & \text { Electric potential, } \mathrm{V} \\ \omega & \text { Angular frequency of the electric field, } \mathrm{rad} / \mathrm{s} \\ \Omega_{0} & \text { Angular spatial period, } \mathrm{rad} / \mathrm{m} \\ \ell & x \text { or } y \text { direction } \\ \nabla & \text { Differential operator, } \nabla=\left(\frac{\partial}{\partial x}, \frac{\partial}{\partial y}\right)\end{array}$

\section{Subscripts}

$\begin{array}{ll}a & \text { Air } \\ d & \text { Dielectric } \\ m & \text { Medium } \\ p & \text { Particle }\end{array}$

455 [1] A. Sayyah, M. N. Horenstein, M. K. Mazumder, Energy yield loss caused by dust deposition on photovoltaic panels, Solar Energy 107 (2014) 576 - 604. 
[2] A. Sayyah, M. Horenstein, M. Mazumder, Mitigation of soiling losses in concentrating solar collectors, in: Photovoltaic Specialists Conference (PVSC), 2013 IEEE 39th, Tampa, FL, 2013, pp. 0480-0485.

[3] M. Mani, R. Pillai, Impact of dust on solar photovoltaic (PV) performance: Research status, challenges and recommendations, Renewable and Sustainable Energy Reviews 14 (9) (2010) 3124 3131.

[4] R. Bkayrat, Developing solutions for the environmental challenges to deploying PV plants in desert areas, Presented in Solar Middle East Conference (Feb 2013).

[5] A. M. Pavan, A. Mellit, D. D. Pieri, The effect of soiling on energy production for large-scale photovoltaic plants, Solar Energy 85 (5) (2011) 1128 - 1136.

[6] J. B. Blackmon, H. H. Dixon, Dust buildup tests of heliostats and mirror specimens, Tech. Rep. MDC-G-7543, McDonnell Douglas Astronautics Co., Huntington Beach, CA (1978).

[7] M. B. Sheratte, Cleaning agents and technologies for concentrating solar collectors, Tech. Rep. 79-7052, Sandia Labs., Albuquerque, NM (1979).

[8] M. Anderson, A. Grandy, J. Hastie, A. Sweezey, R. Ranky, C. Mavroidis, Robotic device for cleaning photovoltaic panel arrays, in: 12th International Conference on Climbing and Walking Robots and the Support Technologies for Mobile Machines, Istanbul, Turkey, 2009, pp. 1-11.

[9] GEKKO Solar Farm: Automated Cleaning Solution for Large Free Field Solar Power Plants (2016). URL http://bit.ly/1TVHJ3A

[10] Heliotex: Automatic Solar Panel Cleaning Systems (2016). URL http: / / bit.ly/1PQfZvu

[11] S. Masuda, K. Fujibayashi, K. Ishida, H. Inaba, Confinement and transportation of charged aerosol clouds via electric curtain, Electrical Engineering in Japan 92 (1) (1972) 43-52.

[12] S. Masuda, Y. Matsumoto, Contact-type electric curtain for electrodynamical control of charged dust particles, in: 2nd International Conference on Static Electricity, no. 72, Frankfurt, Germany, 1973, pp. 1370-1409. 
[13] H. Kawamoto, T. Shibata, Electrostatic cleaning system for removal of sand from solar panels, Journal of Electrostatics 73 (2015) 65 - 70.

[14] M. N. Horenstein, M. K. Mazumder, R. C. Sumner, J. Stark, T. Abuhamed, R. Boxman, Modeling of trajectories in an electrodynamic screen for obtaining maximum particle removal efficiency, Industry Applications, IEEE Transactions on 49 (2) (2013) 707-713.

[15] M. N. Horenstein, M. K. Mazumder, R. C. Sumner, Predicting particle trajectories on an electrodynamic screen - theory and experiment, Journal of Electrostatics 71 (3) (2013) 185-188.

[16] M. Mazumder, M. Horenstein, J. Stark, D. Erickson, A. Sayyah, S. Jung, F. Hao, Development of self-cleaning solar collectors for minimizing energy yield loss caused by dust deposition, in: ASME 2013 7th International Conference on Energy Sustainability collocated with the ASME 2013 Heat Transfer Summer Conference and the ASME 2013 11th International Conference on Fuel Cell Science, Engineering and Technology, American Society of Mechanical Engineers, 2013, pp. V001T11A013-V001T11A013.

[17] M. K. Mazumder, M. N. Horenstein, J. W. Stark, J. N. Hudelson, A. Sayyah, C. Heiling, J. Yellowhair, Electrodynamic removal of dust from solar mirrors and its applications in concentrated solar power (csp) plants, in: Industry Applications Society Annual Meeting, 2014 IEEE, 2014, pp. $1-7$.

[18] M. K. Mazumder, M. N. Horenstein, J. W. Stark, J. N. Hudelson, A. Sayyah, N. Joglekar, J. Yellowhair, A. Botts, Self-cleaning solar mirrors using electrodynamic dust shield: Prospects and progress, in: ASME 2014 8th International Conference on Energy Sustainability collocated with the ASME 2014 12th International Conference on Fuel Cell Science, Engineering and Technology, American Society of Mechanical Engineers, 2014, pp. V001T02A055-V001T02A055.

[19] A. Sayyah, M. Horenstein, M. Mazumder, Performance restoration of dusty photovoltaic modules using electrodynamic screen, in: Photovoltaic Specialist Conference (PVSC), 2015 IEEE 42nd, 2015, pp. 1-3.

[20] M. Mazumder, M. Horenstein, C. Heiling, J. Stark, A. Sayyah, J. Yellowhair, A. Raychowdhury, Environmental degradation of the optical surface of pv modules and solar mirrors by soiling and 
high rh and mitigation methods for minimizing energy yield losses, in: Photovoltaic Specialist Conference (PVSC), 2015 IEEE 42nd, 2015, pp. 1-6.

[21] H. A. Pohl, Dielectrophoresis: the behavior of neutral matter in nonuniform electric fields, Vol. 80, Cambridge University Press, 1978.

[22] J. Kadaksham, P. Singh, N. Aubry, Manipulation of particles using dielectrophoresis, Mechanics Research Communications 33 (1) (2006) 108-122.

[23] C. H. Kua, Y. C. Lam, C. Yang, K. Youcef-Toumi, I. Rodriguez, Modeling of dielectrophoretic force for moving dielectrophoresis electrodes, Journal of Electrostatics 66 (9) (2008) 514-525.

[24] M. Li, W. Li, J. Zhang, G. Alici, W. Wen, A review of microfabrication techniques and dielectrophoretic microdevices for particle manipulation and separation, Journal of Physics D: Applied Physics 47 (6) (2014) 063001.

[25] R. Pethig, Review articledielectrophoresis: status of the theory, technology, and applications, Biomicrofluidics 4 (2) (2010) 022811.

[26] H. Kawamoto, K. Seki, N. Kuromiya, Mechanism of travelling-wave transport of particles, Journal of Physics D: Applied Physics 39 (6) (2006) 1249.

[27] C. Calle, C. Buhler, J. McFall, S. Snyder, Particle removal by electrostatic and dielectrophoretic forces for dust control during lunar exploration missions, Journal of Electrostatics 67 (2) (2009) 89-92.

[28] A. Sayyah, M. N. Horenstein, M. K. Mazumder, A comprehensive analysis of the electric field distribution in an electrodynamic screen, Journal of Electrostatics 76 (2015) 115 - 126.

[29] H. Morgan, N. G. Green, AC electrokinetics: colloids and nanoparticles, no. 2, Research Studies Press, 2003.

[30] M. Washizu, T. B. Jones, Multipolar dielectrophoretic force calculation, Journal of Electrostatics 33 (2) (1994) $187-198$.

[31] T. B. Jones, Electromechanics of particles, Cambridge University Press, 1995. 
[32] K. Engelen, P. Jacqmaer, J. Driesen, Electric and magnetic fields of two infinitely long parallel cylindrical conductors carrying a dc current, The European Physical Journal Applied Physics 64 (02) (2013) 24515.

[33] S. J. Orfanidis, Electromagnetic waves and antennas, Rutgers University New Brunswick, NJ, 2014.

[34] J. A. Cross, Electrostatics: principles, problems and applications, Adam Hilger, 1987.

[35] J. N. Israelachvili, Intermolecular and surface forces, 3rd Edition, Academic press, 2011.

[36] O. H. Pakarinen, A. S. Foster, M. Paajanen, T. Kalinainen, J. Katainen, I. Makkonen, J. Lahtinen, R. M. Nieminen, Towards an accurate description of the capillary force in nanoparticle-surface interactions, Modelling and Simulation in Materials Science and Engineering 13 (7) (2005) 11751186.

[37] A. Çolak, H. Wormeester, H. J. Zandvliet, B. Poelsema, Surface adhesion and its dependence on surface roughness and humidity measured with a flat tip, Applied surface science 258 (18) (2012) 6938-6942.

[38] Y. I. Rabinovich, J. J. Adler, M. S. Esayanur, A. Ata, R. K. Singh, B. M. Moudgil, Capillary forces between surfaces with nanoscale roughness, Advances in colloid and interface science 96 (1) (2002) 213-230.

[39] S. You, M. P. Wan, Mathematical models for the van der waals force and capillary force between a rough particle and surface, Langmuir 29 (29) (2013) 9104-9117. 